\title{
A New 4D Hyperchaotic System and Its Generalized Function Projective Synchronization
}

\author{
Yuan Gao ${ }^{1,2}$ and Chenghua Liang ${ }^{2}$ \\ ${ }^{1}$ School of Information Engineering, Wuhan University of Technology, Wuhan 430070, China \\ ${ }^{2}$ Department of Electronic Information and Control Engineering, Guangxi University of Technology, Liuzhou 545006, China \\ Correspondence should be addressed to Yuan Gao; gxgaoy98@21cn.com
}

Received 21 May 2013; Revised 6 November 2013; Accepted 20 November 2013

Academic Editor: Jun-Juh Yan

Copyright (c) 2013 Y. Gao and C. Liang. This is an open access article distributed under the Creative Commons Attribution License, which permits unrestricted use, distribution, and reproduction in any medium, provided the original work is properly cited.

\begin{abstract}
A new four-dimensional hyperchaotic system is investigated. Numerical and analytical studies are carried out on its basic dynamical properties, such as equilibrium point, Lyapunov exponents, Poincaré maps, and chaotic dynamical behaviors. We verify the realizability of the new system via an electronic circuit by using Multisim software. Furthermore, a generalized function projective synchronization scheme of two different hyperchaotic systems with uncertain parameters is proposed, which includes some existing projective synchronization schemes, such as generalized projection synchronization and function projective synchronization. Based on the Lyapunov stability theory, a controller with parameters update laws is designed to realize synchronization. Using this controller, we realize the synchronization between Chen hyperchaotic system and the new system to verify the validity and feasibility of our method.
\end{abstract}

\section{Introduction}

Chaos is a very fascinating nonlinear phenomenon, which exhibits extreme sensitivity to initial conditions and has noise-like behaviors. Since Lorenz discovered the first chaotic attractor in a three-dimensional (3D) autonomous system in 1963 [1], chaos has been extensively investigated in mathematics, physics, and engineering field. In recent decades, there has been increasing interest in creating the chaos, particularly, since Chen chaotic system is founded by an feedback control (or chaotification) [2]. By now, some typical chaotic systems, such as modified Lorenz system [3], Lü system [4], unified system [5], have been constructed by the consideration of chaotification.

In general, hyperchaotic system is defined as a chaotic system with more than one positive Lyapunov exponent, this implies that its chaotic dynamics extend in several different directions simultaneously. Therefore, comparing with the traditional chaotic system, hyperchaotic system has more complex dynamical behaviors which can be used to enhance the security of chaotic communication system. Consequently, the topic of theoretical design and circuitry realization of various hyperchaotic systems has recently become hotspot in the nonlinear research field. In recent years, hyperchaos has been found numerically and experimentally by adding a simple state feedback controller to Chua's circuit [6], Chen system [7], or Lorenz equation [8].

Inspired by the above works, in this paper, we firstly introduce a new $4 \mathrm{D}$ hyperchaotic system via modifying $3 \mathrm{D}$ Liu system [9]. The basic nonlinear dynamical properties of the new system, such as equilibrium point, Lyapunov exponents, Poincaré maps, and chaotic behaviors, are studied numerically and analytically. Numerical results demonstrate that the new system can exhibit complex hyperchaos over a wide range of system parameters. This hyperchaotic system can be realized by a simple electronic circuit. The Multisim circuit experiment results show good agreement with the numerical simulation. Therefore, the main merit of the proposed hyperchaotic system is suitable as a generator to enhance the security of chaos communication.

On the other hand, in the past decade, chaos synchronization has attracted much more attention for the wide-scope 
potential applications in physics, biology, secure communication, and so forth [10-13]. Since the pioneering work of Pecora and Carroll [14], different types of synchronization have been investigated, such as complete synchronization [15], phase synchronization $[16,17]$, lag synchronization [18], generalized synchronization $[19,20]$, and projective synchronization [2123]. Amongst all the types of chaos synchronization, projective synchronization with proportional feature between the drive system and the response system, first reported by Mainieri and Rehacek in 1999 [21], has aroused more interest because this feature can be used to extend binary digital to M-nary digital communication for achieving faster secure communication [24, 25].

In recent years, inspired by the projective synchronization, increasing attention has been devoted to the problem of the synchronization with more complex feature between the drive system and the response system. $\mathrm{Wu}$ and $\mathrm{Lu}$ [26] studied generalized projective synchronization (GPS) of the fractional-order Chen hyperchaotic system. In [27], a modified projective synchronization (MPS) scheme was investigated, in which the dynamical states of the drive system and response system synchronized up to a constant scaling matrix. Then, Chen et al. [28] proposed a new projective synchronization called function projective synchronization (FPS), in which the drive and response vectors can be synchronized up to a desired scaling function, but not a constant. Therefore, the FPS is very useful to obtain more secure communication, due to the unpredictability of the scaling function which can additionally enhance the security in the communication. In [29], Du et al. introduced a modified function projective synchronization (MFPS) of chaotic system, where the dynamical states of the drive and response system can be synchronized up to a desired scaling function matrix. By now, there have been some research works about the MFPS [29-35]. Particularly, in [36], Yu et al. further proposed a generalized function projective synchronization (GFPS) of two different chaotic systems with fully unknown parameters. The GFPS scheme is an extension of many existing projective synchronization schemes, such as MPS and FPS in which the responses of synchronized dynamical states can be also synchronized up to a scaling function matrix.

From the existing results, one can see that the scaling function matrix in the MFPS is separately considered as a function matrix of drive system variable [30-32,36] or a time function matrix $[29,33-35]$. Obviously, if the combination of two kinds of matrixes is introduced into the synchronization, the scaling function factors become more unpredictable, and better security of chaos communication can be obtained. However, to the best of our knowledge, so far, no research results have been presented about the MFPS where the scaling matrix is composed of time function matrix and system variable function matrix. On the other hand, in the most of practical situations, chaos synchronization can be discussed for two strictly different systems, such as in social science and biological science. Furthermore, the parameters of many systems cannot be known entirely; the synchronization problem of two hyperchaotic systems is more significant for improving the security of communication. Therefore, the above discussions motivate our study.

In this paper, we dedicate to further investigate a new type of GFPS scheme between two different uncertain hyperchaotic systems. In our GFPS scheme, a significant advantage is that the scaling function matrix is constructed by explicit time function matrix and function matrix of drive system variables. Thus, synchronization relationship is more complex. Moreover, complete synchronization, anti-phase synchronization, GPS, and FPS are the special cases of the proposed GFPS. In order to realize the GFPS of two different uncertain hyperchaotic systems, we make an attempt to design a controller with parameters update laws based on Lyapunov stability theory. The GFPS of Chen hyperchaotic system and the proposed hyperchaotic system is considered as an example; the simulation results of this example verify the validity of our method.

This paper is organized as follows. In Section 2, a new $4 \mathrm{D}$ hyperchaotic system is introduced, and its nonlinear dynamical properties and circuit implementation are studied. Furthermore, a GFPS of different hyperchaotic systems with uncertain parameters is investigated in Section 3. The controller for GFPS based on Lyapunov stability theory is put forward, and simulation results of an example are provided to realize the synchronization between Chen hyperchaotic system and the new system in this section. Finally conclusion in Section 4 closes the paper.

\section{The Proposed 4D Dynamic System}

By modifying the 3D Liu chaotic system [9], we introduce the following $4 \mathrm{D}$ quadratic smooth autonomous system:

$$
\begin{gathered}
\dot{x}_{1}=-a_{1} x_{1}+a_{2} x_{2}, \\
\dot{x}_{2}=a_{3} x_{1}-x_{1} x_{3}-x_{2}+x_{4}, \\
\dot{x}_{3}=x_{1}^{2}-a_{4}\left(x_{1}+x_{3}\right), \\
\dot{x}_{4}=-a_{5} x_{1},
\end{gathered}
$$

where $\left(x_{1}, x_{2}, x_{3}, x_{4}\right)^{T} \in R^{4}$ is the state vector and $a_{i}(i=$ $1,2, \ldots, 5)$ are positive constant parameters of the system. The values of the parameters in system (1) are $a_{1}=25$, $a_{2}=60, a_{3}=40, a_{4}=4$, and $a_{5}=5$, so the new system is hyperchaotic in this case. For the purpose to reveal the hyperchaotic dynamics of this new system, in the following paragraphs, some basic properties of dynamic system (1) are analyzed.

2.1. Dissipativity and the Existence of Attractor. The divergence of the system (1) is written by

$$
\nabla V=\frac{\partial \dot{x}_{1}}{\partial x_{1}}+\frac{\partial \dot{x}_{2}}{\partial x_{2}}+\frac{\partial \dot{x}_{3}}{\partial x_{3}}+\frac{\partial \dot{x}_{4}}{\partial x_{4}}=-a_{1}-1-a_{4}<0
$$




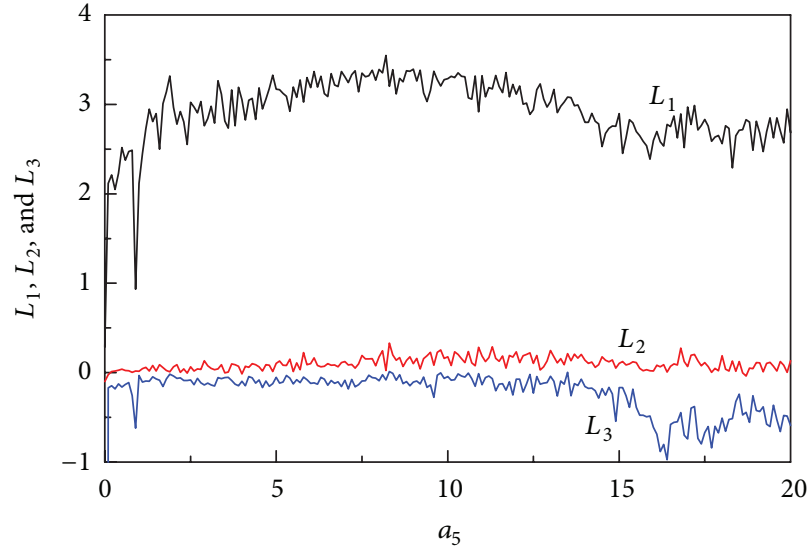

(a)

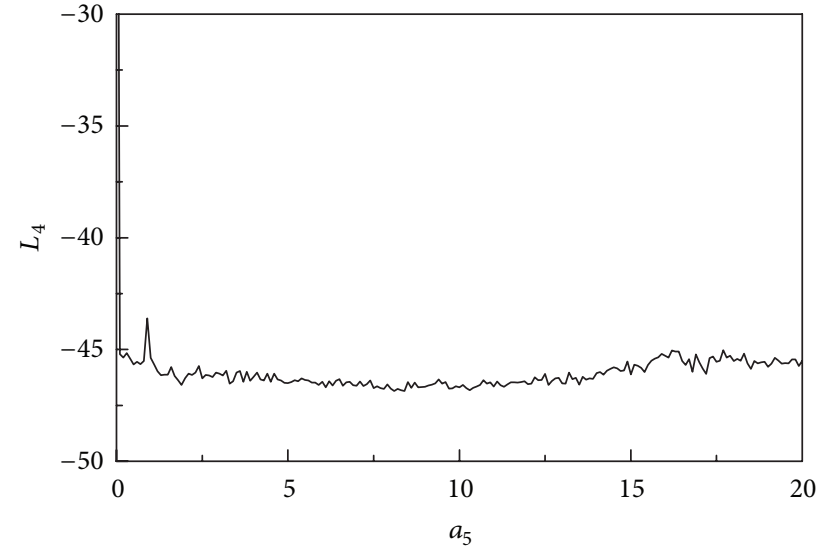

(b)

Figure 1: Lyapunov exponents versus parameter $a_{5}\left(a_{1}=25, a_{2}=60, a_{3}=40\right.$, and $\left.a_{4}=4\right)$.

Therefore, system (1) is dissipative system with an exponential contraction rate $\dot{V}=-\left(a_{1}+a_{4}+1\right) V$. It means that a volume element $V_{0}$ including the system orbit is shrunken to zero as $t \rightarrow \infty$ at an exponential rate $\nabla V$. That is, to say, all the system orbits will finally be restricted to a special subset of zero volume and the asymptotic motion settles onto an attractor of the system (1).

2.2. Equilibrium Point and Stability. The only equilibrium point $O(0,0,0,0)$ of system (1) can be found, while $\dot{x}_{1}=\dot{x}_{2}=$ $\dot{x}_{3}=\dot{x}_{4}=0$. By linearizing the system (1) at $O$, one obtains Jacobian matrix

$$
J=\left[\begin{array}{cccc}
-a_{1} & a_{2} & 0 & 0 \\
a_{3} & -1 & 0 & 1 \\
-a_{4} & 0 & -a_{4} & 0 \\
-a_{5} & 0 & 0 & 0
\end{array}\right] .
$$

Thus, the characteristic equation of the linearized system is expressed as

$$
\begin{aligned}
F(\lambda)= & \lambda^{4}+\left(1+a_{1}+a_{4}\right) \lambda^{3} \\
& +\left(a_{1}-a_{2} a_{3}+a_{1} a_{4}+a_{4}\right) \lambda^{2} \\
& +\left(a_{1} a_{4}+a_{2} a_{5}-a_{2} a_{3} a_{4}\right) \lambda \\
& +a_{2} a_{4} a_{5}=0 .
\end{aligned}
$$

Setting the parameters $a_{1}=25, a_{2}=60, a_{3}=40$, and $a_{4}=4$ and using Routh-Hurwitz criterion to solve (4), one can find two eigenvalues with positive real part at the range of $a_{5}>$ 0 . For example, when $a_{5}=5$, the eigenvalues $\lambda_{1}=-4$, $\lambda_{2}=-63.48, \lambda_{3}=37.35$, and $\lambda_{4}=0.126$, respectively. Characteristic equation has two positive roots and two negative roots, which implies that the fixed point $O(0,0,0,0)$ is an unstable saddle point. While the parameters $a_{1}=25, a_{2}=60$, $a_{4}=4, a_{5}=5$, and $a_{3} \in(0, \infty)$, characteristic equation has also two roots with positive real part. For example, if $a_{3}=10$, the eigenvalues $\lambda_{1}=-4, \lambda_{2}=-40.41, \lambda_{3}=13.87$, $\lambda_{4}=0.534$, respectively. Therefore, $O$ is still an unstable saddle point.
2.3. Lyapunov Spectra and Poincaré Map. The numerical simulations are carried out, by means of Rouge-Kutta integration algorithm, to solve the differential equations. Lyapunov exponents are calculated out by Wolf algorithm [37]. The initial values of system are set to $\left(x_{1}(0), x_{2}(0), x_{3}(0), x_{4}(0)\right)^{T}=$ $(2.0,6.0,9.0,2.0)^{T}$.

When the parameters $a_{1}=25, a_{2}=60, a_{3}=40$, and $a_{4}=4$ are fixed and the parameter $a_{5}$ is varied from zero to 20 , the Lyapunov exponent spectrum of system (1) with respect to $a_{5}$ is shown in Figure 1. Figure 2 indicates the Lyapunov spectrum corresponding the system parameters $a_{1}=25, a_{2}=$ $60, a_{4}=4, a_{5}=5$, and $a_{3} \in[10,50]$. From Figures 1 and 2 , one can see clearly that the $4 \mathrm{D}$ system has two positive Lyapunov exponents over a wide range of parameters $a_{3}$ and $a_{5}$; thus, the presented system is hyperchaotic.

For the parameter values $\left(a_{1}, a_{2}, a_{3}, a_{4}, a_{5}\right)=(25,60$, $40,4,5)$, four Lyapunov exponents of system (1) are calculated as $L_{1}=3.0057, L_{2}=0.0304, L_{3}=-0.1631$, and $L_{4}=$ -46.1578 , respectively. Therefore, the Lyapunov dimension of system (1) is

$$
D=j+\frac{1}{\left|L_{j+1}\right|} \sum_{i=1}^{j} L_{i}=3+\frac{L_{1}+L_{2}+L_{3}}{\left|L_{4}\right|}=3.0622 .
$$

The Lyapunov dimension is fractional, which implies that system (1) is really a dissipative system. In Figures 3(a) and $3(\mathrm{~b})$, we show phase portraits of the orbits on $x_{1}-x_{2}$ and $x_{1}-x_{3}$ planes, it is evident that the system has a double-scroll hyperchaotic attractor. Taking

$$
\begin{aligned}
& \sum_{1}=\left\{\left[x_{1}, x_{2}, x_{3}, x_{4}\right]^{T} \in R^{4} \mid x_{3}=50\right\}, \\
& \sum_{2}=\left\{\left[x_{1}, x_{2}, x_{3}, x_{4}\right]^{T} \in R^{4} \mid x_{2}=0\right\},
\end{aligned}
$$

as cross sections, Poincaré maps on $x_{1}-x_{2}$ and $x_{1}-x_{3}$ planes are illustrated in Figures 4(a) and 4(b), respectively, which show the existence of the chaotic properties in the dynamical system. In the discussion below, the parameters of the new hyperchaotic system $a_{1}=25, a_{2}=60, a_{3}=40, a_{4}=4$, and $a_{5}=5$ are used. 


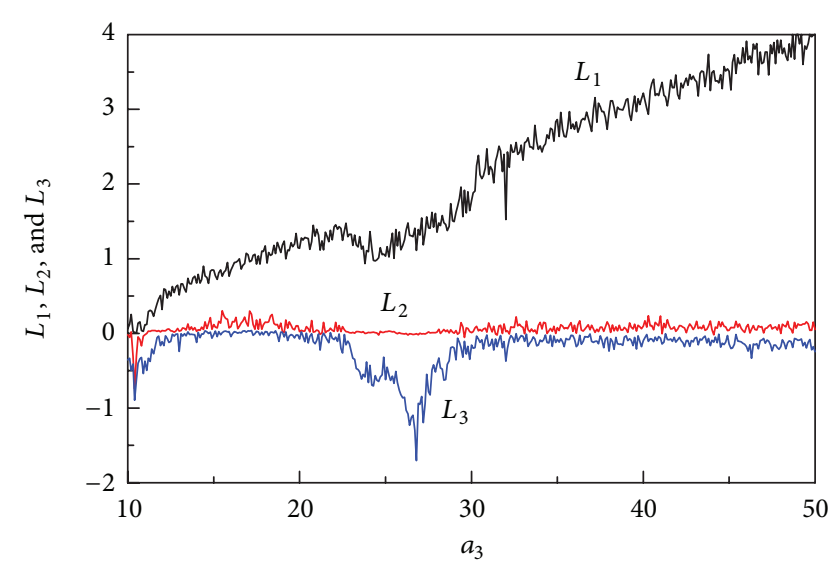

(a)

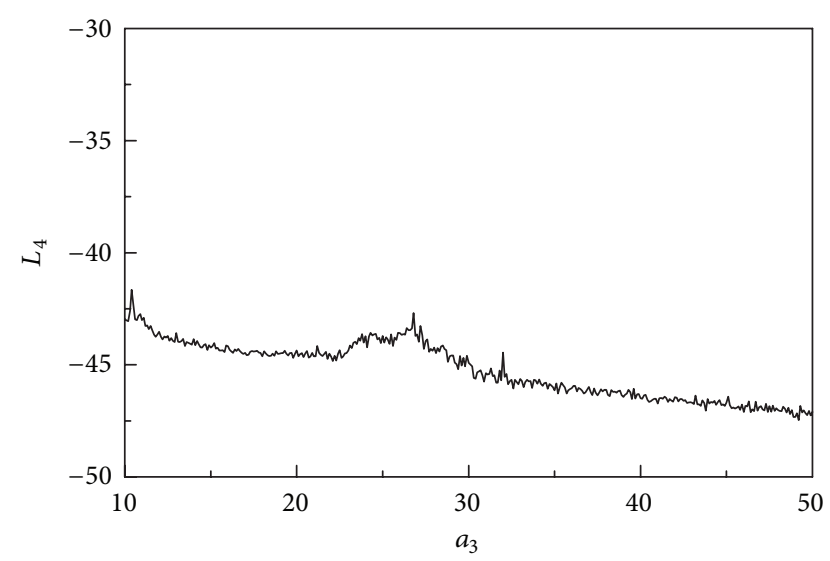

(b)

FIGURE 2: Lyapunov exponents versus parameter $a_{3}\left(a_{1}=25, a_{2}=60, a_{4}=4\right.$, and $\left.a_{5}=5\right)$.

2.4. Verification via Circuit Simulation. In order to verify the realizability of the proposed hyperchaotic system in practical applications, we design an electronic circuit based on the mathematical model (1) with parameters $\left(a_{1}, a_{2}, a_{3}, a_{4}, a_{5}\right)=$ $(25,60,40,4,5)$. By proper choice of the resistors and capacitors, this circuit demonstrates chaotic behavior. In our implementation, output signals of the system states are all compressed to $10 \%$ of theoretical values. The operational amplifiers (TL741 chip) and associated circuitry perform the basic operations of addition, subtraction, and integration. The nonlinear terms in the equation are implemented with the analog multipliers (AD633 chip). Figure 5 gives the circuit schematic of the hyperchaotic system. Based on Kirchhoff's current law, the circuit equations can be written as follows:

$$
\begin{gathered}
\dot{u}_{C_{1}}=-\frac{1}{R_{2} C_{1}} u_{C_{1}}+\frac{R_{10}}{R_{9} R_{1} C_{1}} u_{C_{2}}, \\
\dot{u}_{C_{2}}=\frac{R_{4}}{R_{3} R_{5} C_{2}} u_{C_{1}}-\frac{R_{15}}{10 R_{14} R_{6} C_{2}} u_{C_{1}} u_{C_{3}}-\frac{1}{R_{8} C_{2}} u_{C_{2}} \\
+\frac{R_{18}}{R_{17} R_{7} C_{2}} u_{C_{4}}, \\
\dot{u}_{C_{3}}=-\frac{1}{R_{12} C_{3}} u_{C_{1}}{ }^{2}-\frac{1}{R_{11} C_{3}} u_{C_{1}}-\frac{1}{R_{13} C_{3}} u_{C_{3}}, \\
\dot{u}_{C_{4}}=-\frac{1}{R_{16} C_{4}} u_{C_{1}},
\end{gathered}
$$

where $u_{C_{i}}(i=1,2,3,4)$ are the voltage on the capacitor terminals, which correspond to the system states $x_{i}(i=$ $1,2,3,4)$, respectively. The parameters in the circuit are designed as $R_{1}=16.6667 \mathrm{k} \Omega, R_{2}=40 \mathrm{k} \Omega, R_{5}=25 \mathrm{k} \Omega$, $R_{8}=1000 \mathrm{k} \Omega, R_{16}=200 \mathrm{k} \Omega, R_{11}=R_{13}=250 \mathrm{k} \Omega, R_{6}=$ $R_{7}=R_{12}=100 \mathrm{k} \Omega, R_{3}=R_{4}=R_{9}=R_{10}=R_{14}=R_{15}=$ $R_{17}=R_{18}=10 \mathrm{k} \Omega$, and $C_{1}=C_{2}=C_{3}=C_{4}=1 \mu \mathrm{F}$.

Multisim software is used to simulate the realized circuit of the hyperchaotic system. The chaotic attractors measured by Multisim experiment are shown in Figure 6, which are almost same as the numerical results shown in Figure 3. The comparison between the numerical results and the circuit experimental simulation demonstrates a very good qualitative agreement to each other. Therefore, the functionality of the proposed system is confirmed.

\section{GFPS of Hyperchaotic Systems}

3.1. The Definition of GFPS. From the viewpoint of control, the synchronization task is to design a proper controller which obtains signals from the drive system to tune the behavior of the response system. Here, we consider the hyperchaotic (drive and response) systems as the following form:

$$
\begin{gathered}
\dot{\mathbf{x}}=f(\mathbf{x}), \\
\dot{\mathbf{y}}=g(\mathbf{y})+\mathbf{u}(\mathbf{x}, \mathbf{y}),
\end{gathered}
$$

where $\mathbf{x}=\left(x_{1}, x_{2}, \ldots, x_{n}\right)^{T} \in R^{n}, \mathbf{y}=\left(y_{1}, y_{2}, \ldots, y_{n}\right)^{T} \in R^{n}$ are the state vectors of the systems (8) and (9), respectively, $f, g: R^{n} \rightarrow R^{n}$ are two continuous vector functions, and $\mathbf{u}=\left(u_{1}, u_{2}, \ldots, u_{n}\right)^{T} \in R^{n}$ is a controller which need to be designed.

Definition 1. For the drive system (8) and the unidirectionally coupled response system (9), they are said to be (GFPS) if there exists vector function $\mathbf{u}(\mathbf{x}, \mathbf{y})$ such that

$$
\lim _{t \rightarrow \infty}\|\mathbf{y}-\boldsymbol{\Phi}(t) \mathbf{H}(\mathbf{x}) \mathbf{x}\| \longrightarrow 0
$$

where $\mathbf{H}(\mathbf{x})=\operatorname{diag}\left(h_{1}(\mathbf{x}), h_{2}(\mathbf{x}), \ldots, h_{n}(\mathbf{x})\right), h_{i}(\mathbf{x}): R^{n} \rightarrow R$ are continuous functions, $\boldsymbol{\Phi}(t)=\operatorname{diag}\left(\varphi_{1}(t), \varphi_{2}(t), \ldots, \varphi_{n}(t)\right)$, $\varphi_{i}(t) \neq 0(i=1,2, \ldots, n)$ are continuously differentiable functions with bounded, and $\|\cdot\|$ represents the matrix norm.

Remark 2. The function matrix $\boldsymbol{\Phi}(t) \mathbf{H}(\mathbf{x})$ is called the scaling matrix, and $\varphi_{1}(t) h_{1}(\mathbf{x}), \varphi_{2}(t) h_{2}(\mathbf{x}), \ldots, \varphi_{n}(t) h_{n}(\mathbf{x})$ are called scaling function factors. In the existing results [29-36], the scaling matrix $\boldsymbol{\Phi}(t)$ or $\mathbf{H}(\mathbf{x})$ is always treated separately. 


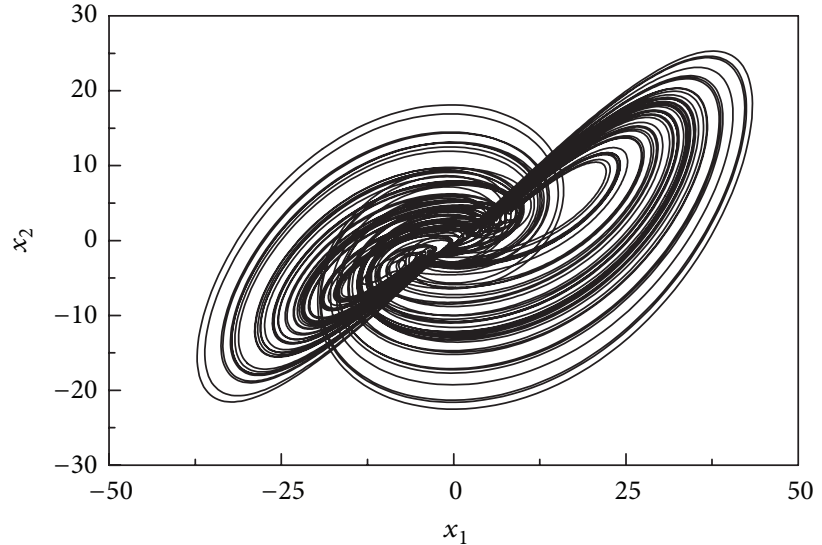

(a) projection on the $x_{1}-x_{2}$ plane

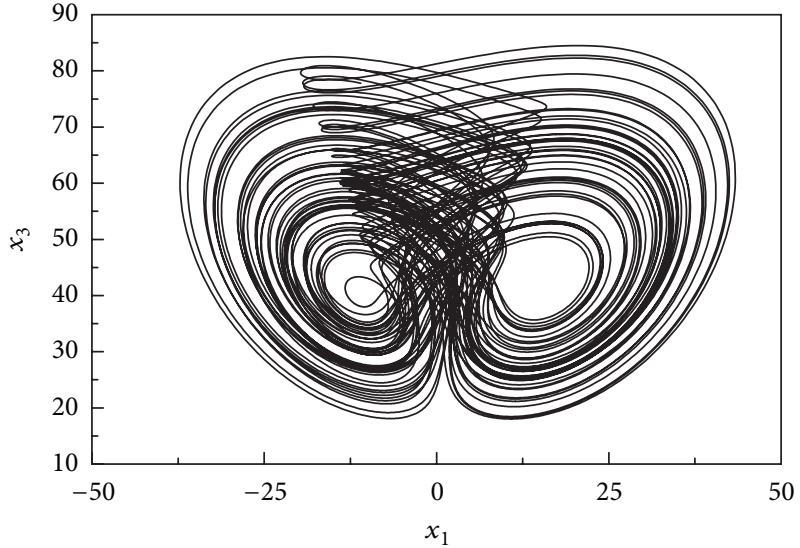

(b) projection on the $x_{1}-x_{3}$ plane

FIGURE 3: Phase portraits of the hyperchaotic attractor $\left(a_{1}=25, a_{2}=60, a_{4}=4, a_{3}=40\right.$, and $\left.a_{5}=5\right)$.

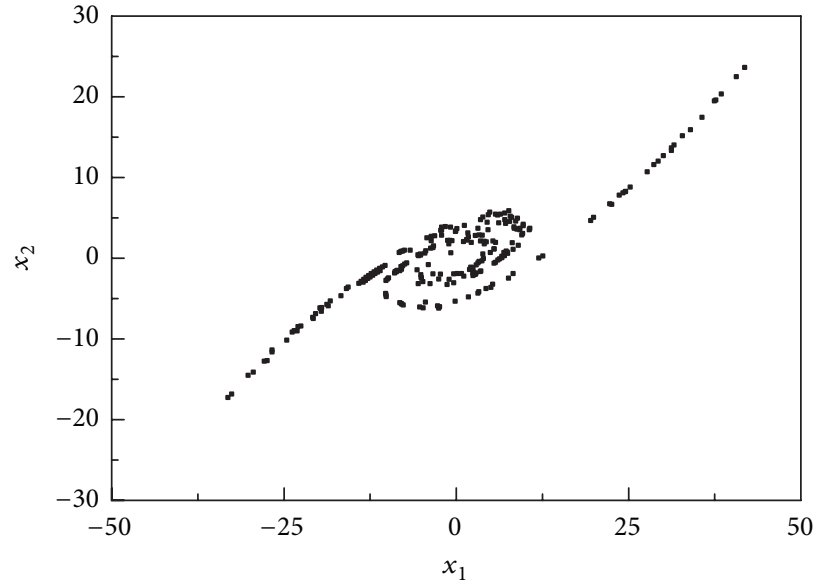

(a) $x_{3}=50$

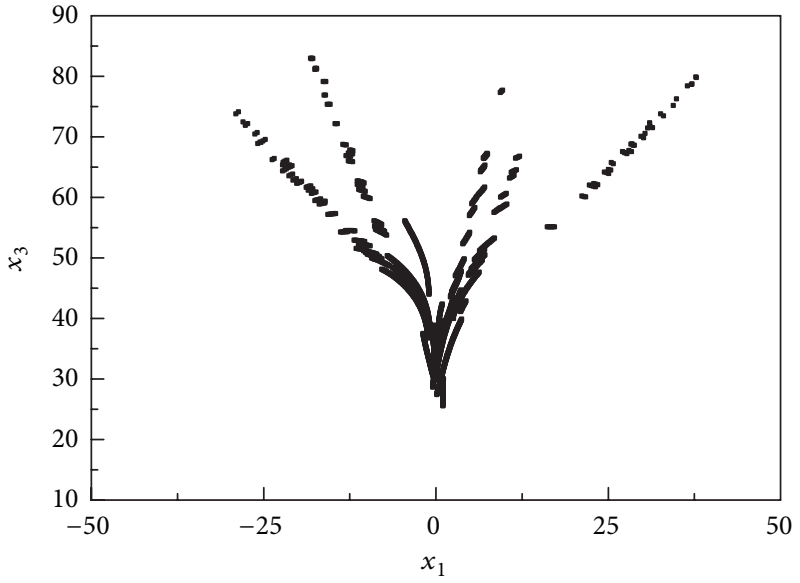

(b) $x_{2}=0$

Figure 4: Poincaré maps of the hyperchaotic attractor on different cross sections $\left(a_{1}=25, a_{2}=60, a_{4}=4, a_{3}=40\right.$, and $\left.a_{5}=5\right)$.

The function matrix in our work is a complex integrated form. Consequently, the presented GFPS can improve antidecryption and unpredictability of the secure communication. Obviously, the GFPS scheme becomes function projective synchronization (FPS) when $\varphi_{1}(t) h_{1}(\mathbf{x})=\varphi_{2}(t) h_{2}(\mathbf{x})=\cdots=$ $\varphi_{n}(t) h_{n}(\mathbf{x})$. Furthermore, while $\varphi_{i}(t) h_{i}(\mathbf{x})(i=1,2, \ldots, n)$ are constants, GFPS will be simplified into generalized projective synchronization (GPS). In particular, the problem is further reduced to the complete synchronization and anti-phase synchronization if $\Phi(t) \mathbf{H}(\mathbf{x})=I$ and $\boldsymbol{\Phi}(t) \mathbf{H}(\mathbf{x})=-I$, where $I$ is an $n \times n$ identity matrix.

Remark 3. The error vector for GFPS between the drive system (8) and the response system (9) is defined by

$$
\mathbf{e}(t)=\mathbf{y}-\boldsymbol{\Phi}(t) \mathbf{H}(\mathbf{x}) \mathbf{x},
$$

where $\mathbf{e}(t)=\left(e_{1}(t), e_{2}(t), \ldots, e_{n}(t)\right)^{T} \in R^{n}$, and $e_{i}=y_{i}-$ $\varphi_{i}(t) h_{i}(\mathbf{x}) x_{i},(i=1,2, \ldots, n)$.
Remark 4. If $\boldsymbol{\Phi}(t) \mathbf{H}(\mathbf{x})=0$, the synchronization problem turns into a chaos control problem.

3.2. The Scheme of GFPS with Unknown System Parameters. Considering GFPS of two different hyperchaotic systems with fully unknown parameters, we can rewrite the drive and response systems (8), (9) as

$$
\begin{gathered}
\dot{\mathbf{x}}=f_{1}(\mathbf{x})+f_{2}(\mathbf{x}) \boldsymbol{\alpha}, \\
\dot{\mathbf{y}}=g_{1}(\mathbf{y})+g_{2}(\mathbf{y}) \boldsymbol{\beta}+\mathbf{u},
\end{gathered}
$$

respectively, where $f_{1}, g_{1}: R^{n} \rightarrow R^{n}$ are continuous vector functions, $f_{2}: R^{n} \rightarrow R^{n \times m}, g_{2}: R^{n} \rightarrow R^{n \times l}$ are continuous matrix functions, and $\boldsymbol{\alpha} \in R^{m}, \boldsymbol{\beta} \in R^{l}$ are unknown parameter vectors of systems (12) and (13). We introduce $\widetilde{\boldsymbol{\alpha}}$ and $\widetilde{\boldsymbol{\beta}}$ as the 


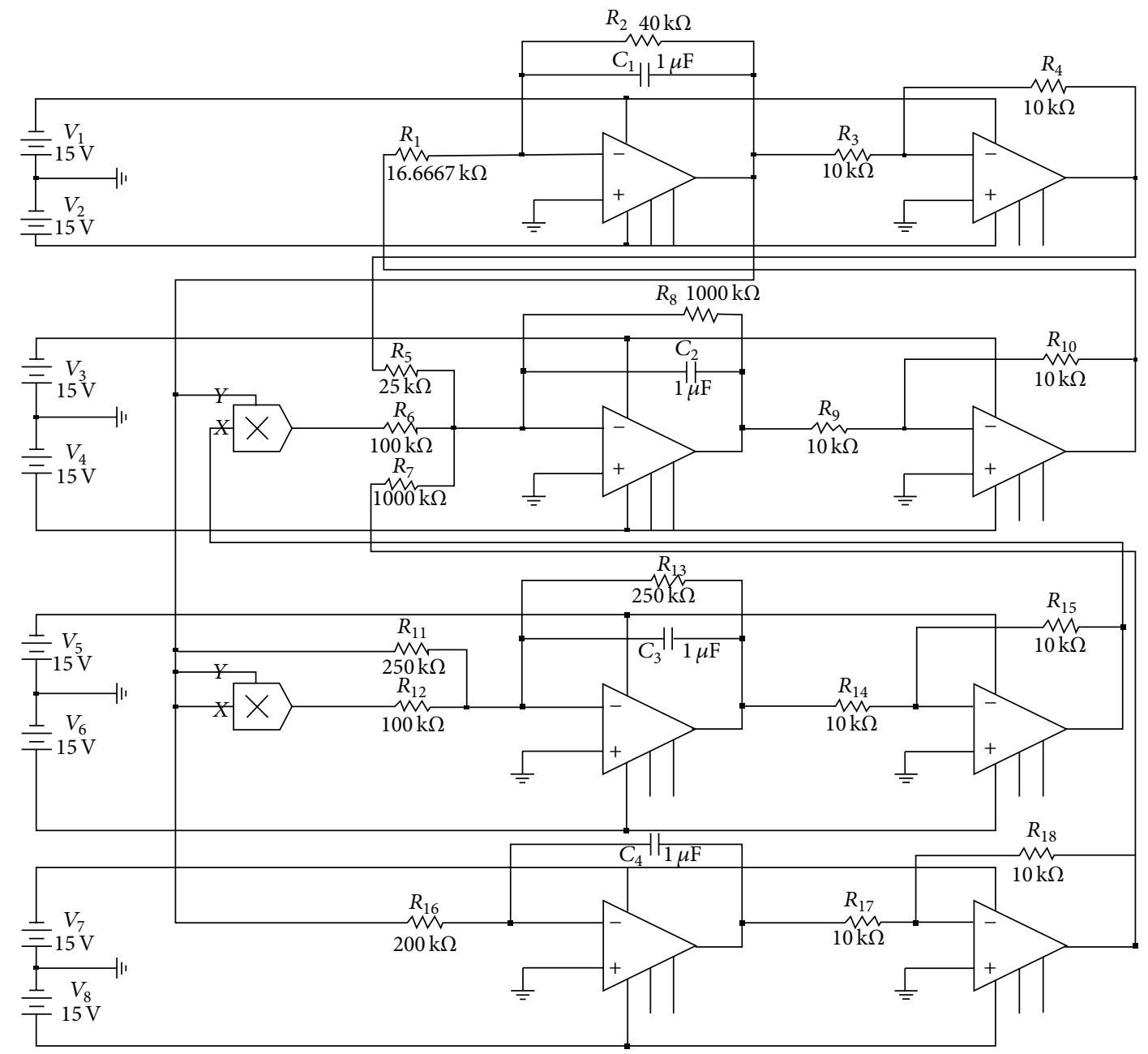

Figure 5: The circuit schematic of the new hyperchaotic system (1).

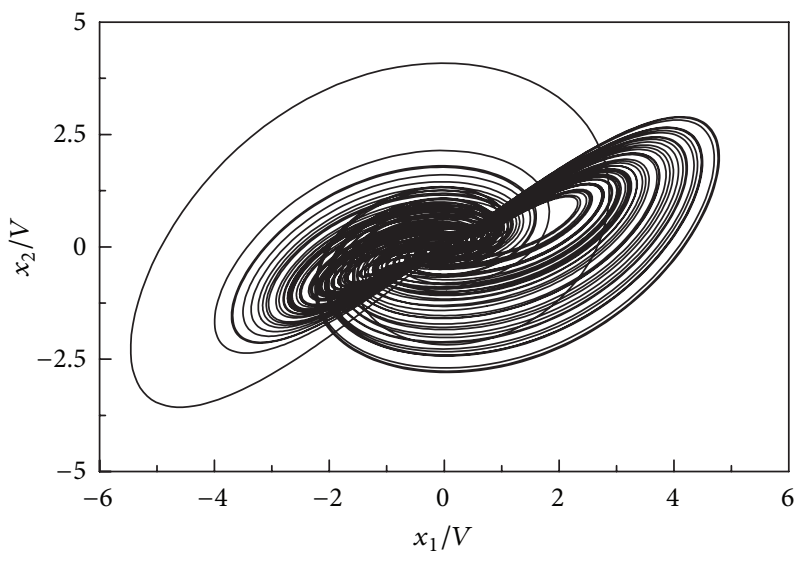

(a)

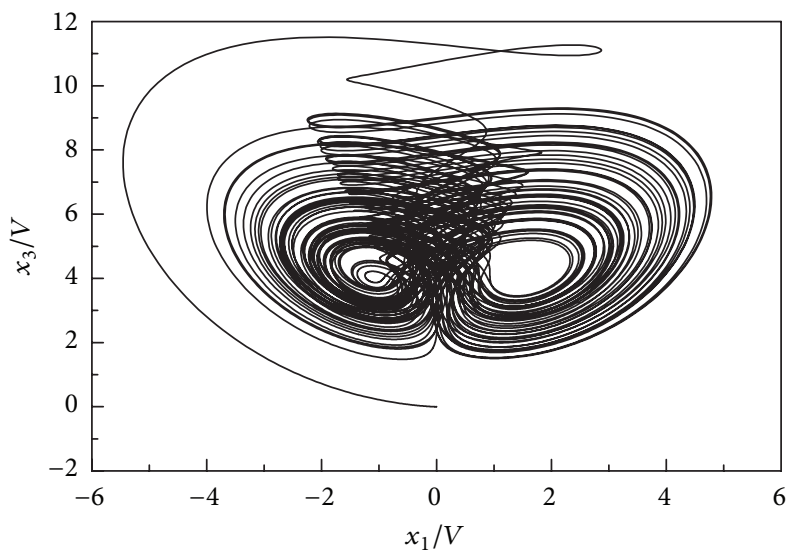

(b)

FIGURE 6: Phase portraits of the hyperchaotic attractor by Multisim experiment. 


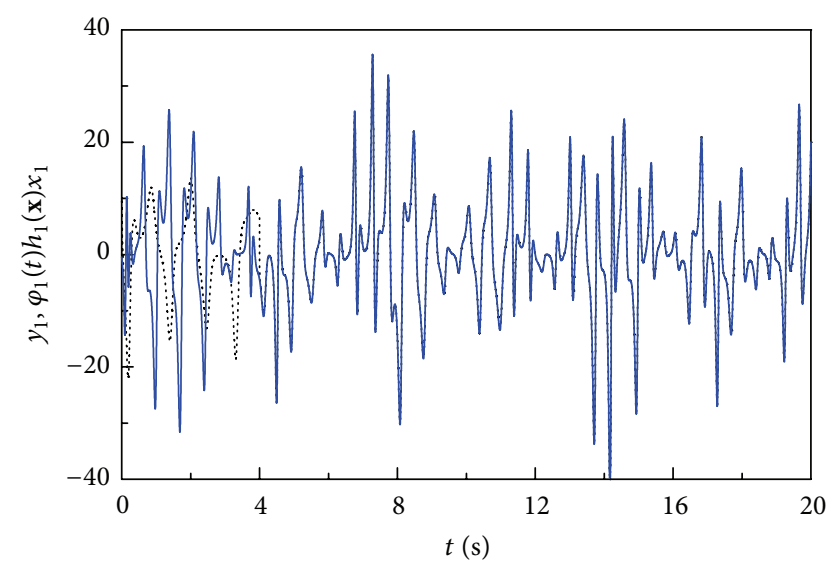

…... $y_{1}$

$\varphi_{1}(t) h_{1}(\mathbf{x}) x_{1}$

(a)

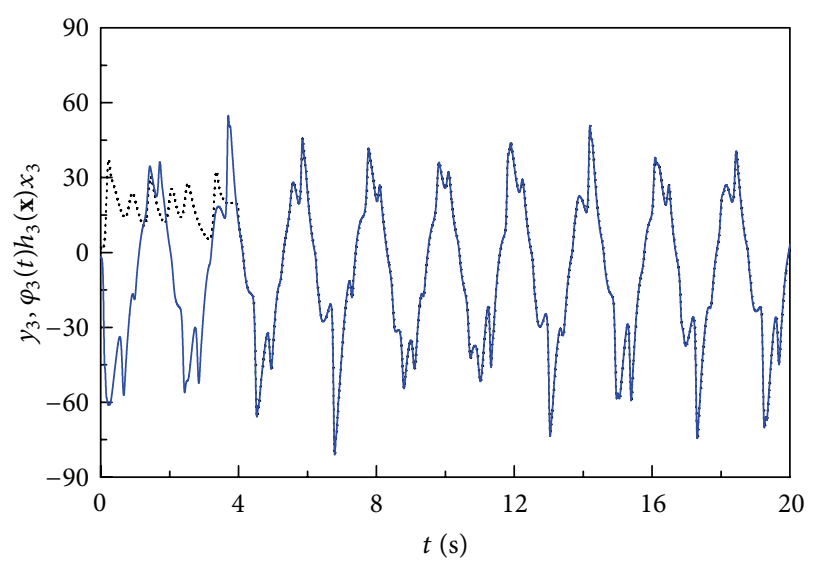

…... $y_{3}$

$-\varphi_{3}(t) h_{3}(\mathbf{x}) x_{3}$

(c)

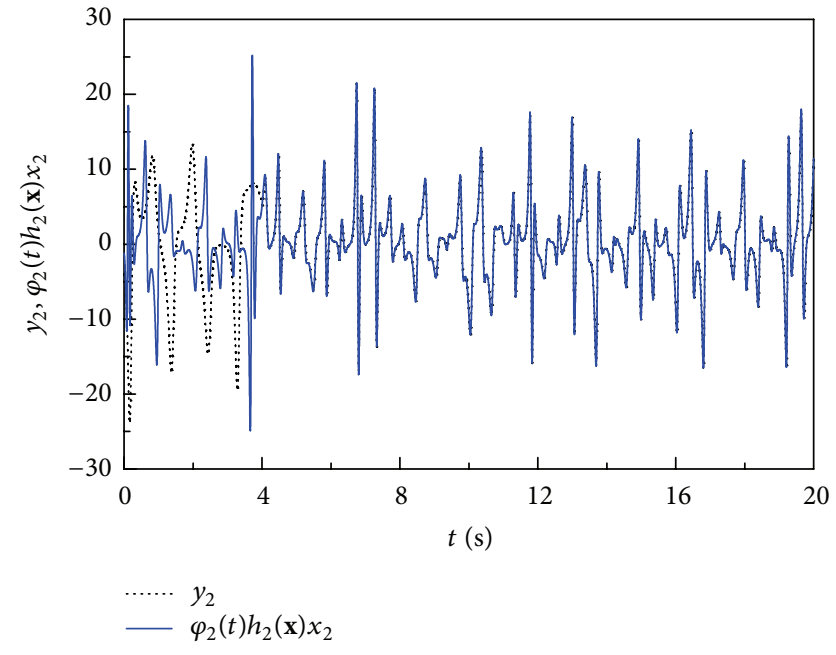

(b)

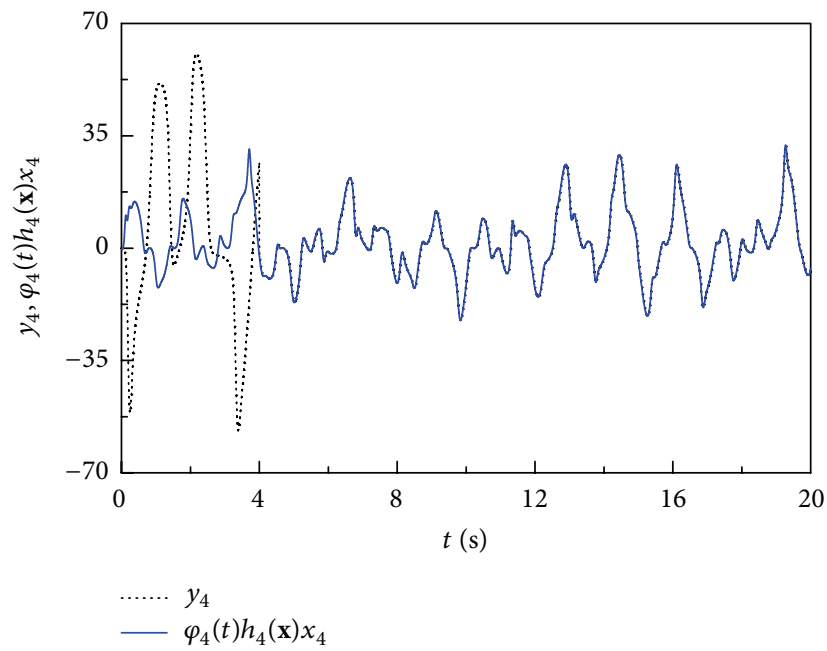

(d)

FIgURE 7: The state trajectories of the drive and response systems $\left(\varphi_{i}(t) h\left(x_{i}\right) x_{i}=\left(c_{i 1} \sin \omega t+c_{i 2}\right)\left(d_{i 1} x_{i}+d_{i 2}\right) x_{i}, c_{i j}=0.1, d_{i 1}=0.02\right.$, $d_{i 2}=-10,(i=1,2,3,4 ; j=1,2)$, and $\left.\left(\omega_{1}, \omega_{2}, \omega_{3}, \omega_{4}\right)=(1,2,3,4)\right)$.

parameter estimation vectors of $\boldsymbol{\alpha}$ and $\boldsymbol{\beta}$, respectively, and define the Jacobian matrix of vector $\mathbf{H}(\mathbf{x}) \mathbf{x}$ as follows:

$$
\Gamma=\frac{d(\mathbf{H}(\mathbf{x}) \mathbf{x})}{d \mathbf{x}} .
$$

Theorem 5. For a given continuous scaling matrix function $\Phi(t) \mathbf{H}(\mathbf{x})$ and all initial conditions $\mathbf{x}(0), \mathbf{y}(0)$, the GFPS between the drive system (12) and the response system (13) can be achieved and the unknown parameters $\boldsymbol{\alpha}$ and $\boldsymbol{\beta}$ can be identified by the following controller and the adaptive parameter update laws:

$$
\begin{gathered}
\mathbf{u}(t)=-g_{1}(\mathbf{y})-g_{2}(\mathbf{y}) \tilde{\boldsymbol{\beta}}+\dot{\Phi}(t) \mathbf{H}(\mathbf{x}) \mathbf{x} \\
+\boldsymbol{\Phi}(t) \mathbf{\Gamma}\left[f_{1}(\mathbf{x})+f_{2}(\mathbf{x}) \tilde{\boldsymbol{\alpha}}\right]-\mathbf{K e}(t), \\
\dot{\tilde{\boldsymbol{\alpha}}}=-f_{2}^{T}(\mathbf{x}) \boldsymbol{\Gamma}^{T} \mathbf{\Phi}(t) \mathbf{e}(t)-\mathbf{P} \mathbf{e}_{\alpha}, \\
\dot{\tilde{\boldsymbol{\beta}}}=g_{2}^{T}(\mathbf{y}) \mathbf{e}(t)-\mathbf{Q} \mathbf{e}_{\beta},
\end{gathered}
$$

respectively, where $\mathbf{K}=\operatorname{diag}\left(k_{1}, k_{2}, \ldots, k_{n}\right), \mathbf{P}=\operatorname{diag}\left(k_{n+1}\right.$, $\left.k_{n+2}, \ldots, k_{n+m}\right), \mathbf{Q}=\operatorname{diag}\left(k_{n+m+1}, k_{n+m+2}, \ldots, k_{n+m+l}\right), k_{i}>$ $0(i=1,2, \ldots, n+m+l)$, and $\mathbf{e}_{\alpha}=\widetilde{\boldsymbol{\alpha}}-\boldsymbol{\alpha}$ and $\mathbf{e}_{\beta}=\widetilde{\boldsymbol{\beta}}-\boldsymbol{\beta}$ are error vectors of the identified parameters.

Proof. The time derivative of the error vector (11) is

$$
\dot{\mathbf{e}}(t)=\dot{\mathbf{y}}-\dot{\Phi}(t) \mathbf{H}(\mathbf{x}) \mathbf{x}-\dot{\Phi}(t) \Gamma \dot{\mathbf{x}} .
$$

Substituting (15) into (17), we have

$$
\begin{aligned}
\dot{\mathbf{e}}(t) & =-g_{2}(\mathbf{y})(\widetilde{\boldsymbol{\beta}}-\boldsymbol{\beta})+\boldsymbol{\Phi}(t) \boldsymbol{\Gamma} f_{2}(\mathbf{x})(\widetilde{\boldsymbol{\alpha}}-\boldsymbol{\alpha})-\mathbf{K e}(t) \\
& =-g_{2}(\mathbf{y}) \mathbf{e}_{\beta}+\boldsymbol{\Phi}(t) \boldsymbol{\Gamma} f_{2}(\mathbf{x}) \mathbf{e}_{\alpha}-\mathbf{K e}(t) .
\end{aligned}
$$




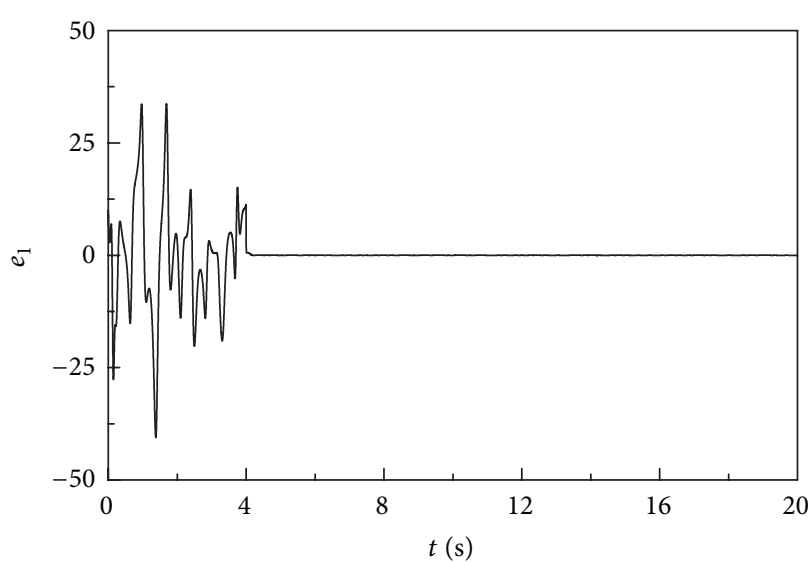

(a)

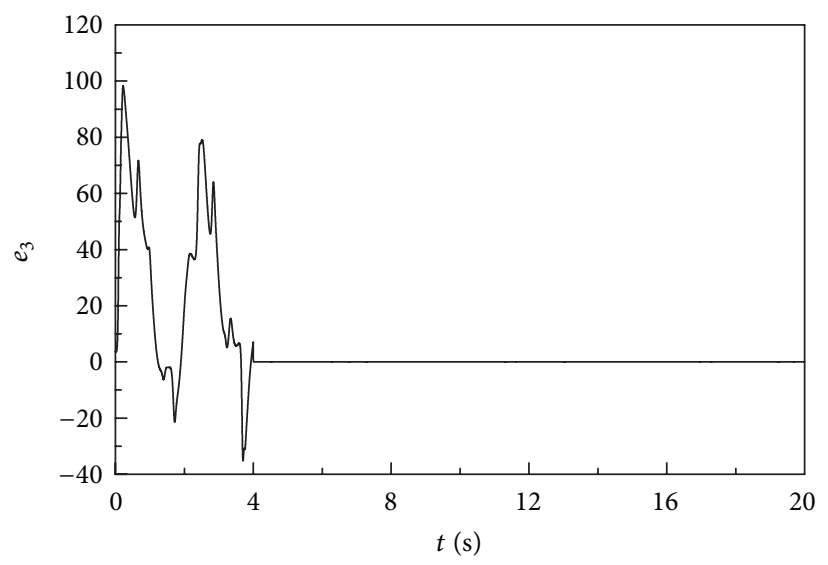

(c)

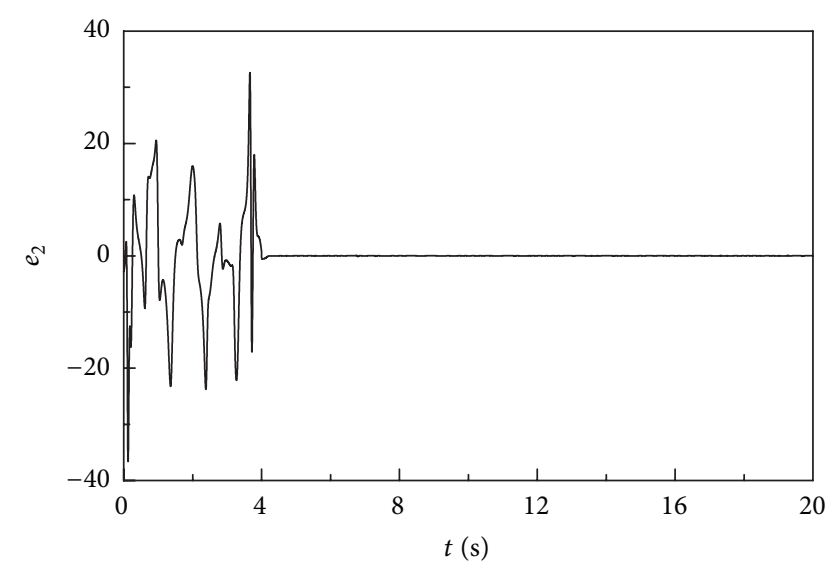

(b)

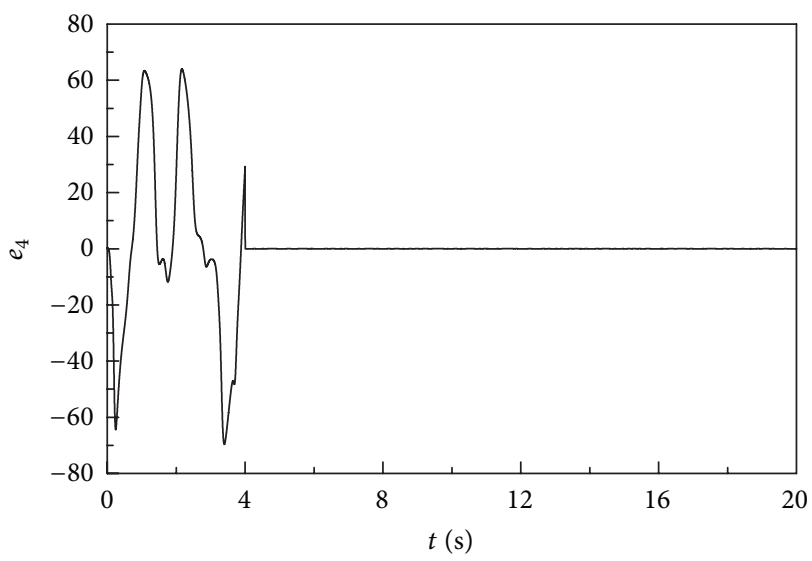

(d)

FIGURE 8: Error curves of GFPS $\left(e_{i}=y_{i}-\varphi_{i}(t) h\left(x_{i}\right) x_{i}, i=1,2,3,4\right)$.

Here, we define the Lyapunov function as

$$
V(t)=\frac{1}{2}\left[\mathbf{e}^{T}(t) \mathbf{e}(t)+\mathbf{e}_{\alpha}^{T}(t) \mathbf{e}_{\alpha}(t)+\mathbf{e}_{\beta}^{T}(t) \mathbf{e}_{\beta}(t)\right] .
$$

From (16) and (18), the first derivative of (19) with respect to time is

$$
\begin{aligned}
\dot{V}(t)= & \mathbf{e}^{T} \dot{\mathbf{e}}+\mathbf{e}_{\alpha}^{T} \dot{\mathbf{e}}_{\alpha}+\mathbf{e}_{\beta}^{T} \dot{\mathbf{e}}_{\beta} \\
= & \mathbf{e}^{T}\left[-g_{2}(\mathbf{y}) \mathbf{e}_{\beta}+\boldsymbol{\Phi}(t) \boldsymbol{\Gamma} f_{2}(\mathbf{x}) \mathbf{e}_{\alpha}-\mathbf{K e}(t)\right] \\
& +\mathbf{e}_{\alpha}^{T}\left[-f_{2}^{T}(\mathbf{x}) \boldsymbol{\Gamma}^{T} \boldsymbol{\Phi}(t) \mathbf{e}(t) \mathbf{P} \mathbf{e}_{\alpha}\right] \\
& +\mathbf{e}_{\beta}^{T}\left[g_{2}^{T}(\mathbf{y}) \mathbf{e}(t)-\mathbf{Q} \mathbf{e}_{\beta}\right] \\
= & -\mathbf{e}^{T}(t) \mathbf{K e}(t)-\mathbf{e}_{\alpha}^{T}(t) \mathbf{P} \mathbf{e}_{\alpha}(t)-\mathbf{e}_{\beta}^{T}(t) \mathbf{Q} \mathbf{e}_{\beta}(t)<0 .
\end{aligned}
$$

According to the Lyapunov stability theorem, the trivial solution of the error system (17) is asymptotically stable, which implies that the GFPS of system (12) and (13) is obtained, and error vectors of parameters $\mathbf{e}_{\alpha}, \mathbf{e}_{\beta}$ approach zero as $t \rightarrow \infty$. The proof is complete.
3.3. Applications. In order to verify the effectiveness and feasibility of the proposed GFPS scheme, we apply the controller to realize the synchronization between Chen hyperchaotic system and the proposed system. Chen hyperchaotic system is described by the following state equations [38]:

$$
\begin{gathered}
\dot{y}_{1}=b_{1}\left(y_{2}-y_{1}\right)+y_{4}, \\
\dot{y}_{2}=b_{2} y_{1}-y_{1} y_{3}+b_{3} y_{2}, \\
\dot{y}_{3}=y_{1} y_{2}-b_{4} y_{3}, \\
\dot{y}_{4}=y_{2} y_{3}+b_{5} y_{4},
\end{gathered}
$$

where $\mathbf{y}=\left(y_{1}, y_{2}, y_{3}, y_{4}\right)^{T}$ is the state vector and $\boldsymbol{\beta}=$ $\left(b_{1}, b_{2}, b_{3}, b_{4}, b_{5}\right)^{T}$ is parameter vector. As the parameters $\left(b_{1}, b_{2}, b_{3}, b_{4}, b_{5}\right)=(35,7,12,3,0.5)$, Chen system shows hyperchaotic properties.

We take the proposed system and Chen hyperchaotic system as the drive system and the response system, respectively. Thus, the parameter vector of the drive system (1) is defined 


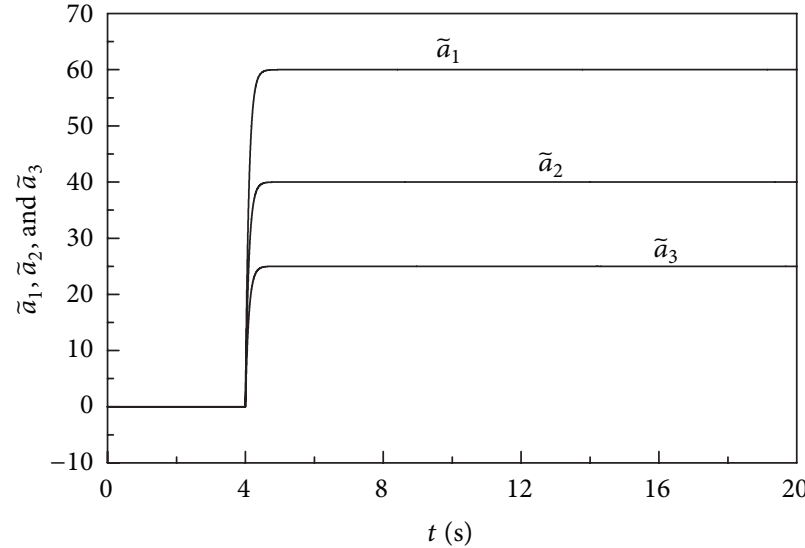

(a)

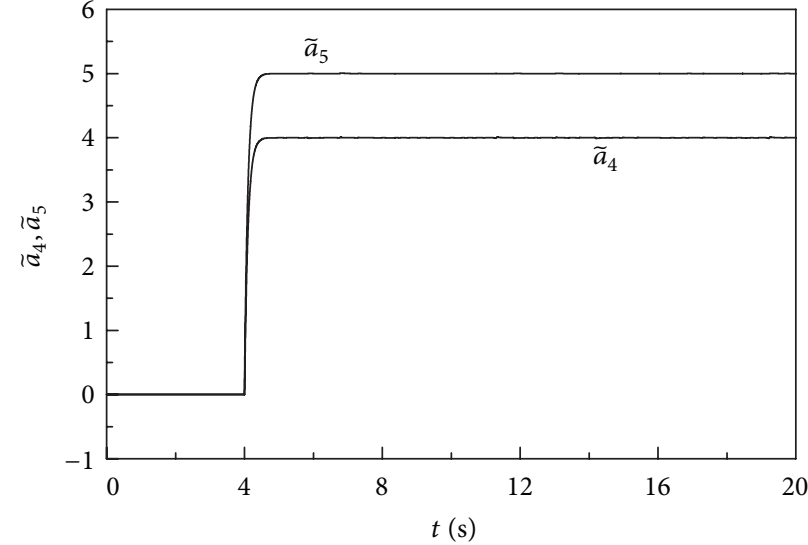

(b)

FigURE 9: The estimated values of the unknown parameters $a_{i}(i=1,2,3,4,5)$ for drive system with true parameters $\left(a_{1}, a_{2}, a_{3}, a_{4}, a_{5}\right)=$ $(25,60,40,4,5)$.

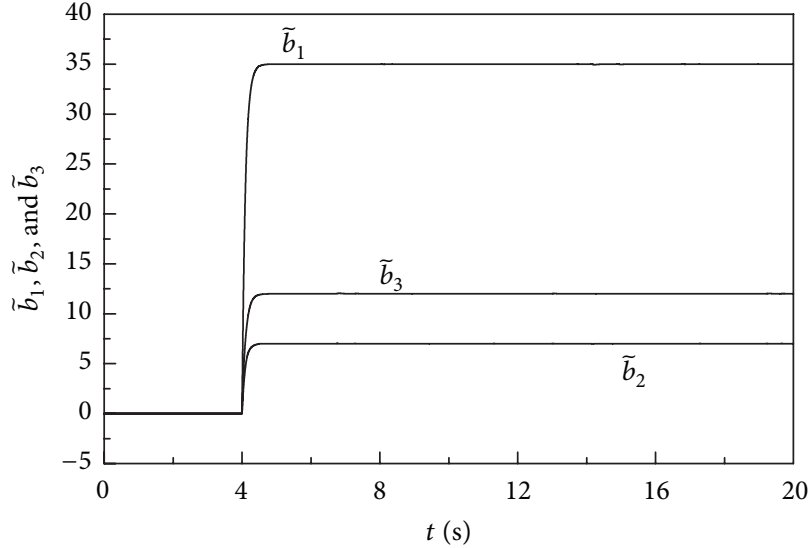

(a)

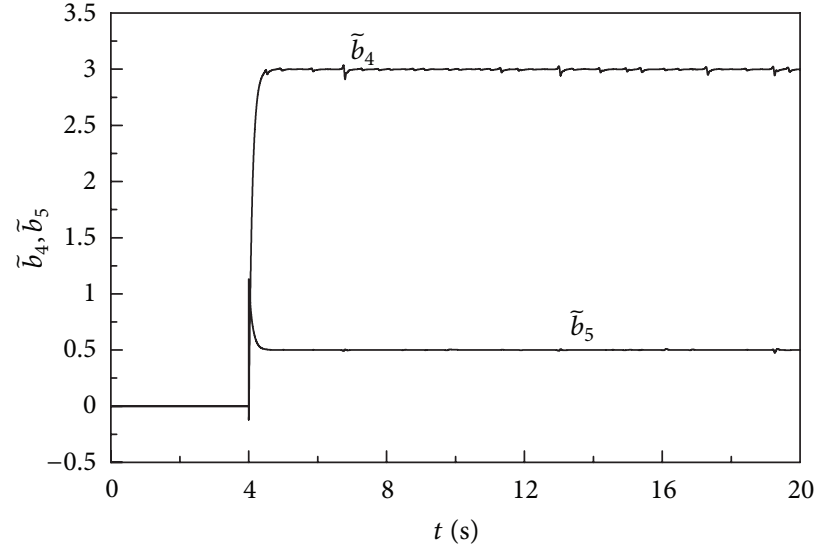

(b)

FIGURE 10: The estimated values of the unknown parameters $b_{i}(i=1,2,3,4,5)$ for response system with true parameters $\left(b_{1}, b_{2}, b_{3}, b_{4}, b_{5}\right)=$ $(35,7,12,3,0.5)$.

as $\boldsymbol{\alpha}=\left(a_{1}, a_{2}, a_{3}, a_{4}, a_{5}\right)^{T}$. The response system can be written as

$$
\begin{gathered}
\dot{y}_{1}=b_{1}\left(y_{2}-y_{1}\right)+y_{4}+u_{1}, \\
\dot{y}_{2}=b_{2} y_{1}+y_{1} y_{3}+b_{3} y_{2}+u_{2}, \\
\dot{y}_{3}=y_{1} y_{2}-b_{4} y_{3}+u_{3}, \\
\dot{y}_{4}=y_{2} y_{3}-b_{5} y_{4}+u_{4},
\end{gathered}
$$

where $u_{1}, u_{2}, u_{3}$, and $u_{4}$ are the control functions to be designed. Without loss of generality, we consider the continuous function matrixes

$$
\begin{aligned}
\boldsymbol{\Phi}(t)= & \operatorname{diag}\left\{\varphi_{1}(t), \varphi_{2}(t), \varphi_{3}(t), \varphi_{4}(t)\right\} \\
= & \operatorname{diag}\left\{c_{11} \sin \omega_{1} t+c_{12}, c_{21} \sin \omega_{2} t+c_{22},\right. \\
& \left.c_{31} \sin \omega_{3} t+c_{32}, c_{41} \sin \omega_{4} t+c_{42}\right\},
\end{aligned}
$$

$$
\begin{array}{r}
\mathbf{H}(\mathbf{x})=\operatorname{diag}\left\{h_{1}(\mathbf{x}), h_{2}(\mathbf{x}), h_{3}(\mathbf{x}), h_{4}(\mathbf{x})\right\} \\
=\operatorname{diag}\left\{d_{11} x_{1}+d_{12}, d_{21} x_{2}+d_{22},\right. \\
\left.d_{31} x_{3}+d_{32}, d_{41} x_{4}+d_{42}\right\},
\end{array}
$$

where $c_{i j}, d_{i j}(i=1,2,3,4 ; j=1,2)$ are constants. According to the GPFS scheme presented above, the synchronization errors are defined as

$$
\begin{aligned}
e_{i}= & y_{i}-\varphi_{i}(t) h_{i}(x) x_{i} \\
= & y_{i}-\left(c_{i 1} \sin \omega_{i} t+c_{i 2}\right) \\
& \quad \times\left(d_{i 1} x_{i}+d_{i 2}\right) x_{i}, \quad i=1,2,3,4 .
\end{aligned}
$$




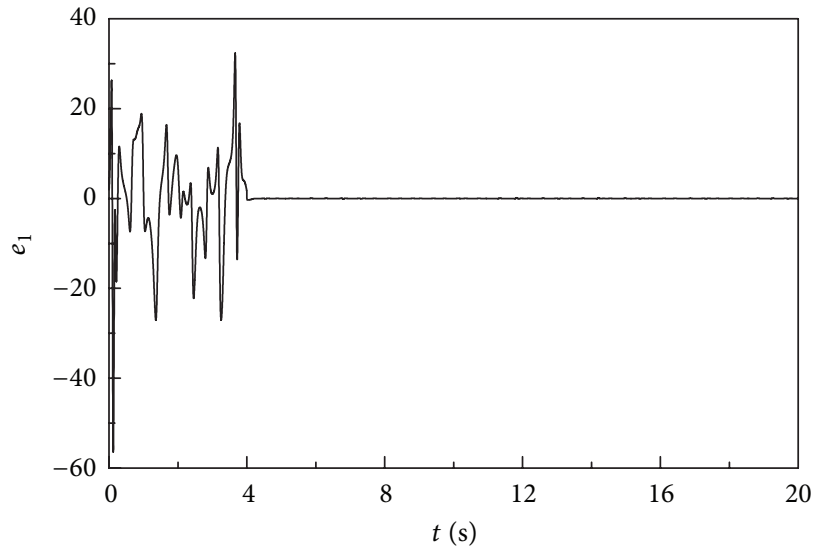

(a)

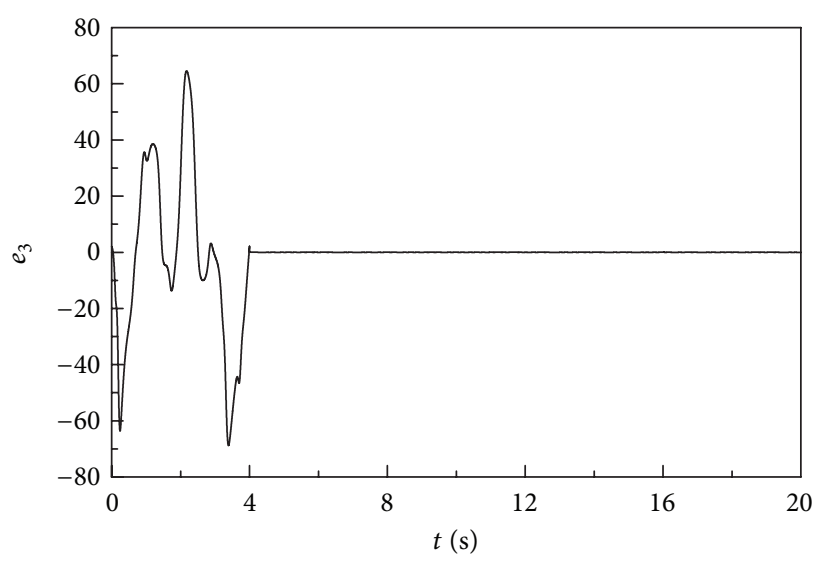

(c)

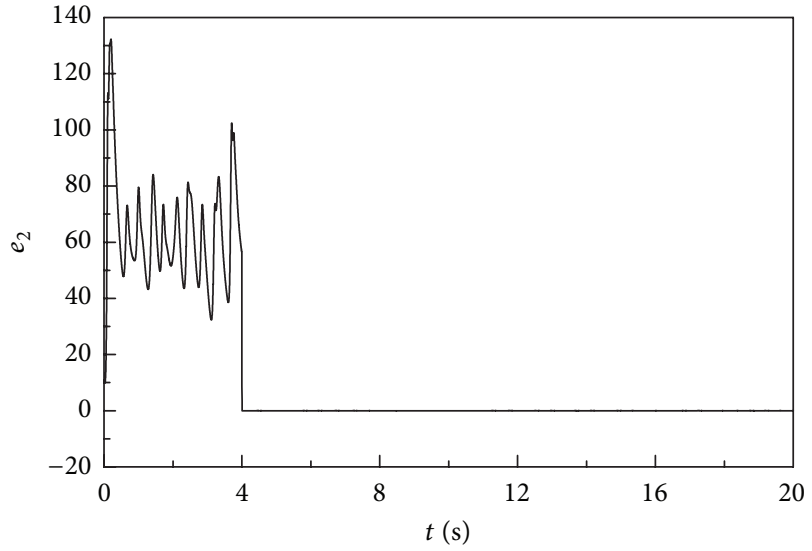

(b)

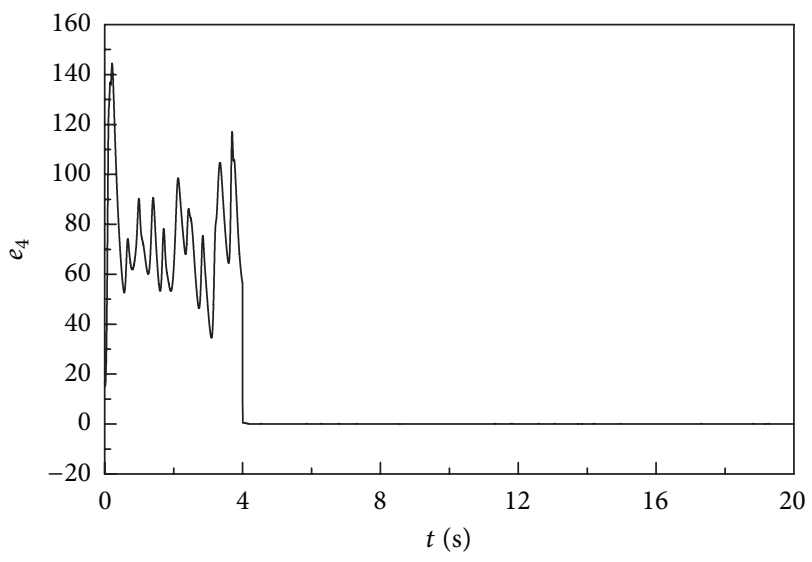

(d)

FIGURE 11: The evolution of anti-phase synchronization error $\left(e_{i}=y_{i}+x_{i}, i=1,2,3,4\right)$.

Substituting (1) and (22) into (25), one can obtain the time derivative of error

$$
\begin{aligned}
\dot{e}_{1}= & y_{4}+b_{1}\left(y_{2}-y_{1}\right)-c_{11} \omega_{1} \cos \omega_{1} t \\
& \times\left(d_{11} x_{1}+d_{12}\right) x_{1}-\left(c_{11} \sin \omega_{1} t+c_{12}\right) \\
& \times\left(2 d_{11} x_{1}+d_{12}\right)\left(-a_{1} x_{1}+a_{2} x_{2}\right)+u_{1}, \\
\dot{e}_{2}= & b_{2} y_{1}+b_{3} y_{2}-y_{1} y_{3}-c_{21} \omega_{2} \cos \omega_{2} t \\
& \times\left(d_{21} x_{2}+d_{22}\right) x_{2}-\left(c_{21} \sin \omega_{2} t+c_{22}\right) \\
& \times\left(2 d_{21} x_{2}+d_{22}\right)\left(a_{3} x_{1}-x_{1} x_{3}-x_{2}+x_{4}\right)+u_{2}, \\
\dot{e}_{3}= & y_{1} y_{2}-b_{4} y_{3}-c_{31} \omega_{3} \cos \omega_{3} t \\
& \times\left(d_{31} x_{3}+d_{32}\right) x_{3}-\left(c_{31} \sin \omega_{3} t+c_{32}\right) \\
& \times\left(2 d_{31} x_{3}+d_{32}\right)\left[x_{1}^{2}-a_{4}\left(x_{1}+x_{3}\right)\right]+u_{3}, \\
\dot{e}_{4}= & y_{2} y_{3}+b_{5} y_{4}-c_{41} \omega_{4} \cos \omega_{4} t\left(d_{41} x_{4}+d_{42}\right) x_{4} \\
& +a_{5} x_{1}\left(c_{41} \sin \omega_{4} t+c_{42}\right)\left(2 d_{41} x_{4}+d_{42}\right)+u_{4} .
\end{aligned}
$$

From (15) and (16), we get the controller

$$
\begin{aligned}
u_{1}= & -y_{4}-\tilde{b}_{1}\left(y_{2}-y_{1}\right)-c_{11} \omega_{1} \cos \omega_{1} t \\
& \times\left(d_{11} x_{1}+d_{12}\right) x_{1}+\left(c_{11} \sin \omega_{1} t+c_{12}\right) \\
& \times\left(2 d_{11} x_{1}+d_{12}\right)\left(-\tilde{a}_{1} x_{1}+\tilde{a}_{2} x_{2}\right)-k_{1} e_{1}, \\
u_{2}= & y_{1} y_{3}-\widetilde{b}_{2} y_{1}-\widetilde{b}_{3} y_{2}+c_{21} \omega_{2} \cos \omega_{2} t \\
& \times\left(d_{21} x_{2}+d_{22}\right) x_{2}+\left(c_{21} \sin \omega_{2} t+c_{22}\right) \\
& \times\left(2 d_{21} x_{1}+d_{22}\right)\left(\tilde{a}_{3} x_{1}-x_{1} x_{3}-x_{2}+x_{4}\right)-k_{2} e_{2}, \\
u_{2}= & \tilde{b}_{4} y_{3}-y_{1} y_{2}+c_{31} \omega_{3} \cos \omega_{3} t \\
& \times\left(d_{31} x_{3}+d_{32}\right) x_{3}+\left(c_{31} \sin \omega_{3} t+c_{32}\right) \\
& \times\left(2 d_{31} x_{1}+d_{32}\right)\left[x_{1} x_{2}-\tilde{a}_{4}\left(x_{1}+x_{3}\right)\right]-k_{3} e_{3}, \\
u_{4}= & -y_{2} y_{3}-\tilde{b}_{5} y_{4}+c_{41} \omega_{4} \cos \omega_{4} t\left(d_{41} x_{4}+d_{42}\right) x_{4} \\
& -\tilde{a}_{5} x_{1}\left(c_{41} \sin \omega_{4} t+c_{42}\right)\left(2 d_{41} x_{4}+d_{42}\right)-k_{4} e_{4},
\end{aligned}
$$




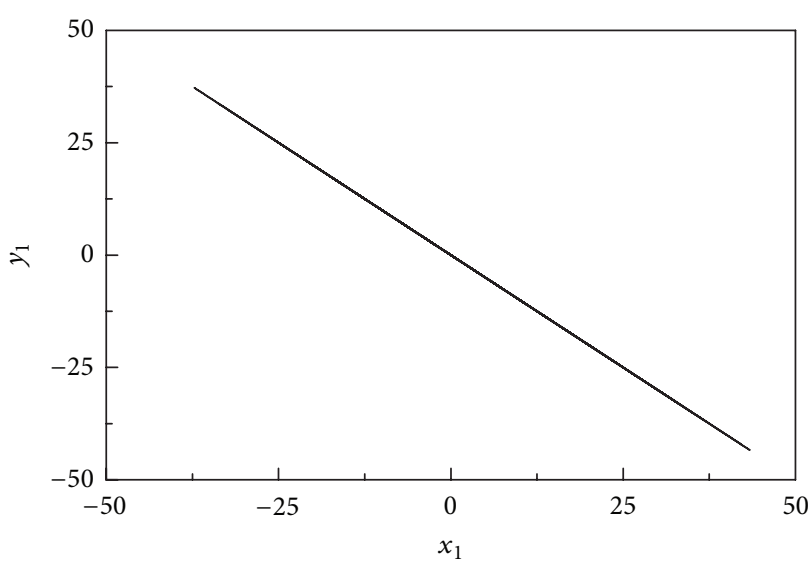

(a)

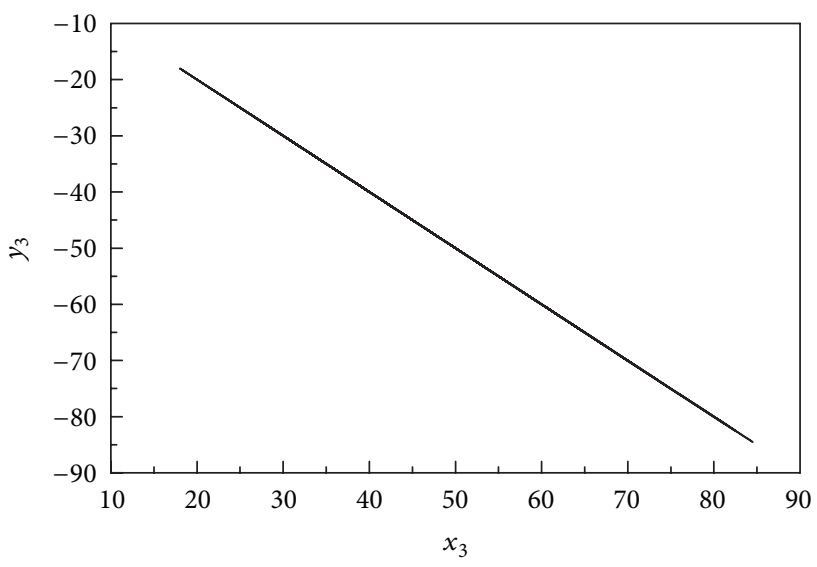

(c)

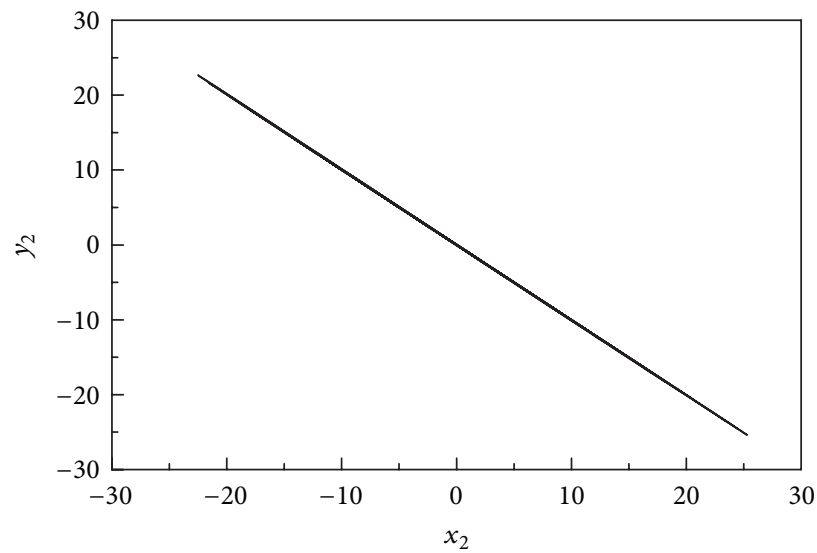

(b)

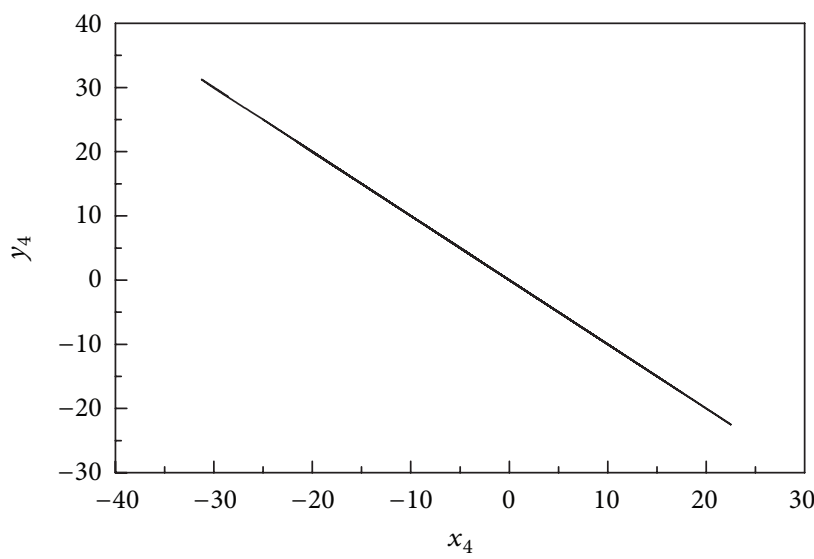

(d)

FIGURE 12: Anti-phase synchronization with $y_{i}=-x_{i}(i=1,2,3,4)$.

and the adaptive parameter update laws

$$
\begin{aligned}
& \dot{\tilde{a}}_{1}=x_{1}\left(c_{11} \sin \omega_{1} t+c_{12}\right)\left(2 d_{11} x_{1}+d_{12}\right) e_{1}-k_{5} e_{a_{1}}, \\
& \dot{\tilde{a}}_{2}=-x_{2}\left(c_{11} \sin \omega_{1} t+c_{12}\right)\left(2 d_{11} x_{1}+d_{12}\right) e_{1}-k_{6} e_{a_{2}}, \\
& \dot{\tilde{a}}_{3}=-x_{1}\left(c_{21} \sin \omega_{2} t+c_{22}\right)\left(2 d_{21} x_{2}+d_{22}\right) e_{2}-k_{7} e_{a_{3}}, \\
& \dot{\tilde{a}}_{4}=\left(x_{1}+x_{5}\right)\left(c_{31} \sin \omega_{3} t+c_{32}\right) \\
& \quad \quad \times\left(2 d_{31} x_{3}+d_{32}\right) e_{3}-k_{8} e_{a_{4}}, \\
& \dot{\tilde{a}}_{5}=x_{1}\left(c_{41} \sin \omega_{4} t+c_{42}\right)\left(2 d_{41} x_{4}+d_{42}\right) e_{4}-k_{9} e_{a_{5}}, \\
& \dot{\tilde{b}}_{1}=\left(y_{2}-y_{1}\right) e_{1}-k_{10} e_{b_{1}}, \\
& \dot{\tilde{b}}_{2}=y_{1} e_{2}-k_{11} e_{b_{2}}, \\
& \dot{\tilde{b}}_{3}=y_{2} e_{2}-k_{12} e_{b_{3}}, \\
& \dot{\tilde{b}}_{4}=-y_{3} e_{3}-k_{12} e_{b_{4}}, \\
& \dot{\tilde{b}}_{5}=y_{4} e_{4}-k_{14} e_{b_{5}},
\end{aligned}
$$

where the control gains $k_{i}>0(i=1,2, \ldots, 14), e_{a_{i}}=\tilde{a}_{i}-$ $a_{i}(i=1,2,3,4,5)$ and $e_{b_{i}}=\widetilde{b}_{i}-b_{i}(i=1,2,3,4,5)$, are the corresponding errors of the identified parameters.

3.4. Simulation Results. In the simulation, the initial values of the drive and response system are set to be $\left(x_{1}(0), x_{2}(0), x_{3}(0), x_{4}(0)\right)=(2.0,6.0,9.0,2.0)$ and $\left(y_{1}(0)\right.$, $\left.y_{2}(0), y_{3}(0), y_{4}(0)\right)=(3.0,-4.0,2.0,2.0)$, respectively. For the simulation purpose, we select all the gains $k_{i}=70(i=$ $1,2, \ldots, 14)$. The true values of "unknown" parameters of new system and Chen system are arbitrarily taken as $\widetilde{a}_{1}=\widetilde{a}_{2}=$ $\widetilde{a}_{3}=\widetilde{a}_{4}=\widetilde{a}_{5}=0$ and $\widetilde{b}_{1}=\widetilde{b}_{2}=\widetilde{b}_{3}=\widetilde{b}_{4}=\widetilde{b}_{5}=0$, respectively. In order to indicate the effectiveness of the controller (27), the control input is applied to the response system after $t=4 \mathrm{~s}$.

We choose the coefficients of the scaling function matrix $\Phi(t) \mathbf{H}(\mathbf{x})$ according to (24) and (25) as $c_{i j}=0.1, d_{i 1}=0.02$, $d_{i 2}=-10(i=1,2,3,4 ; j=1,2)$, and $\left(\omega_{1}, \omega_{2}, \omega_{3}, \omega_{4}\right)=$ $(1,2,3,4)$. Figure 7 provides the trajectories of GFPS between Chen system (22) and new system (1). Synchronization error curves are displayed in Figure 8. From Figures 7 and 
8 , one can see that the following mathematic relationships,

$$
\begin{aligned}
y_{i} & =\varphi_{i}(t) h_{i}(x) x_{i} \\
& =\left(c_{i 1} \sin \omega_{i} t+c_{i 2}\right)\left(d_{i 1} x_{i}+d_{i 2}\right) x_{i}, \quad i=1,2,3,4
\end{aligned}
$$

are satisfied rapidly after control; it means that the GFPS is achieved. The estimated values of unknown parameters for the drive and response system are shown by Figures 9 and 10, respectively. From Figures 9 and 10, it is obvious that the unknown parameters are converged to the true values quickly by applying the parameters update laws.

When $c_{i 1}=d_{i 1}=0, c_{i 2}=-d_{i 2}=1(i=1,2,3,4)$, the scaling functions are simplified as $\varphi_{i}(t) h_{i}(\mathbf{x})=-1(i=$ $1,2,3,4)$. Under the same conditions of the system parameters, the initial statuses, and the control gains, the simulation is operated. Figure 11 displays the time evolutions of antiphase synchronization errors $e_{i}=y_{i}+x_{i}(i=1,2,3,4)$, and it is obvious that $e_{i}(i=1,2,3,4)$ are nonzero and irregular before control and converge to zero rapidly after control. Figure 12 exhibits the synchronization relationship $y_{i}=-x_{i}(i=1,2,3,4)$, which means that anti-phase synchronization between the drive system (1) and the response system (22) is gained.

From the above simulation results of the GPFS, we can find that the synchronization error can be quickly converged to zero, and the unknown parameters of the drive system and the response system all are accurately estimated to true values. This means that our GPFS algorithm based on Lyapunov stability theory is convergent and effective.

\section{Conclusion}

In this paper, a new 4D hyperchaotic system is introduced at first, and some basic nonlinear dynamical properties of the system are studied. The new system shows complex hyperchaotical behaviors over a wide range of system parameters and can be realized by a simple electronic circuit. At this point, this new hyperchaotic system is suitable as a chaotic sequences generator in the secure communication field. Secondly, a new type of GFPS scheme of two different uncertain hyperchaotic systems is investigated. In our case, the scaling matrixes $\boldsymbol{\Phi}(t)$ and $\mathbf{H}(\mathbf{x})$ are no more treated separately but as an integrated form; thus, synchronization relationship is more complex. It should be noted that complete synchronization, anti-phase synchronization, general projective synchronization, and function projective synchronization are the special cases of the presented GFPS. A controller with parameters update laws is designed based on Lyapunov stability theory. The GFPS of Chen hyperchaotic system and the proposed hyperchaotic system is considered as an example; the simulation results of the example verify the validity of our method.

\section{Acknowledgments}

This work was supported by the Natural Science Foundation of Guangxi Province of China (Grant no.
2013GXNSFAA019351) and the Scientific Research Fund of Guangxi Education Department (Grant no. 201202ZD068 and 201204LX657).

\section{References}

[1] E. N. Lorenz, "Deterministic nonperiodic flow," Journal of the Atmospheric Sciences, vol. 20, pp. 130-141, 1963.

[2] G. Chen and T. Ueta, "Yet another chaotic attractor," International Journal of Bifurcation and Chaos, vol. 9, no. 7, pp. 14651466, 1999.

[3] J. Wang, M. Zhao, Y. Zhang, and X. Xiong, "Si'lnokov-type orbits of lorenz-family systems," Physica A, vol. 37, pp. 438-446, 2007.

[4] J. Lü and G. Chen, "A new chaotic attractor coined," International Journal of Bifurcation and Chaos, vol. 12, no. 3, pp. 659661, 2002.

[5] J. Lü, G. Chen, D. Cheng, and S. Celikovsky, "Bridge the gap between the lorenz system and the chen system," International Journal of Bifurcation and Chaos, vol. 12, no. 12, pp. 2917-2926, 2002.

[6] K. Thamilmaran, M. Lakshmanan, and A. Venkatesan, "Hyperchaos in a modified canonical chua's circuit," International Journal of Bifurcation and Chaos, vol. 14, no. 1, pp. 221-243, 2004.

[7] Y. Li, W. K. S. Tang, and G. Chen, "Generating hyperchaos via state feedback control," International Journal of Bifurcation and Chaos, vol. 15, no. 10, pp. 3367-3375, 2005.

[8] Q. Jia, "Hyperchaos generated from the lorenz chaotic system and its control," Physics Letters A, vol. 366, no. 3, pp. 217-222, 2007.

[9] C. Liu, T. Liu, L. Liu, and K. Liu, "A new chaotic attractor," Chaos, Solitons \& Fractals, vol. 22, no. 5, pp. 1031-1038, 2004.

[10] L. Kocarev and U. Parlitz, "General approach for chaotic synchronization with applications to communication," Physical Review Letters, vol. 74, no. 25, pp. 5028-5031, 1995.

[11] H. R. Karimi and P. Maass, "Delay-range-dependent exponential $H_{\infty}$ synchronization of a class of delayed neural networks," Chaos, Solitons \& Fractals, vol. 41, no. 3, pp. 1125-1135, 2009.

[12] B. Nana, P. Woafo, and S. Domngang, "Chaotic synchronization with experimental application to secure communications," Communications in Nonlinear Science and Numerical Simulation, vol. 14, no. 5, pp. 2266-2276, 2009.

[13] H. R. Karimi, "Adaptive $H_{\infty}$ synchronization of master-slave systems with mixed time-varying delays and nonlinear perturbations: an LMI approach," International Journal of Automation and Computing, vol. 8, no. 4, pp. 381-390, 2011.

[14] L. M. Pecora and T. L. Carroll, "Synchronization in chaotic systems," Physical Review Letters, vol. 64, no. 8, pp. 821-824, 1990.

[15] Y. Yu and S. Zhang, "Adaptive backstepping synchronization of uncertain chaotic system," Chaos, Solitons \& Fractals, vol. 21, no. 3, pp. 643-649, 2004.

[16] E.-H. Park, M. A. Zaks, and J. Kurths, "Phase synchronization in the forced lorenz system," Physical Review E, vol. 60, no. 6 A, pp. 6627-6638, 1999.

[17] E. Rosa Jr., E. Ott, and M. H. Hess, "Transition to phase synchronization of chaos," Physical Review Letters, vol. 80, no. 8, pp. 1642-1645, 1998.

[18] M. G. Rosenblum, A. S. Pikovsky, and J. Kurths, "From phase to lag synchronization in coupled chaotic oscillators," Physical Review Letters, vol. 78, no. 22, pp. 4193-4196, 1997. 
[19] N. F. Rulkov, M. M. Sushchik, L. S. Tsimring, and H. D. I. Abarbanel, "Generalized synchronization of chaos in directionally coupled chaotic systems," Physical Review E, vol. 51, no. 2, pp. 980-994, 1995.

[20] Y.-W. Wang and Z.-H. Guan, "Generalized synchronization of continuous chaotic system," Chaos, Solitons \& Fractals, vol. 27, no. 1, pp. 97-101, 2006.

[21] R. Mainieri and J. Rehacek, "Projective synchronization in three-dimensional chaotic systems," Physical Review Letters, vol. 82, no. 15, pp. 3042-3045, 1999.

[22] M. Hu, Z. Xu, R. Zhang, and A. Hu, "Adaptive full state hybrid projective synchronization of chaotic systems with the same and different order," Physics Letters A, vol. 365, no. 4, pp. 315-327, 2007.

[23] J. H. Park, "Adaptive control for modified projective synchronization of a four-dimensional chaotic system with uncertain parameters," Journal of Computational and Applied Mathematics, vol. 213, no. 1, pp. 288-293, 2008.

[24] Z. Li and D. Xu, "A secure communication scheme using projective chaos synchronization," Chaos, Solitons \& Fractals, vol. 22, no. 2, pp. 477-481, 2004.

[25] F. Farivar, M. A. Shoorehdeli, M. A. Nekoui, and M. Teshnehlab, "Generalized projective synchronization of uncertain chaotic systems with external disturbance," Expert Systems with Applications, vol. 38, no. 5, pp. 4714-4726, 2011.

[26] X. Wu and Y. Lu, "Generalized projective synchronization of the fractional-order chen hyperchaotic system," Nonlinear Dynamics, vol. 57, no. 1-2, pp. 25-35, 2009.

[27] G.-H. Li, "Modified projective synchronization of chaotic system," Chaos, Solitons \& Fractals, vol. 32, no. 5, pp. 1786-1790, 2007.

[28] Y. Chen, H. An, and Z. Li, "The function cascade synchronization approach with uncertain parameters or not for hyperchaotic systems," Applied Mathematics and Computation, vol. 197, no. 1, pp. 96-110, 2008.

[29] H. Du, Q. Zeng, and C. Wang, "Modified function projective synchronization of chaotic system," Chaos, Solitons \& Fractals, vol. 42, no. 4, pp. 2399-2404, 2009.

[30] Y. Chen and X. Li, "Function projective synchronization between two identical chaotic systems," International Journal of Modern Physics C, vol. 18, no. 5, pp. 883-888, 2007.

[31] L. Runzi and W. Zhengmin, "Adaptive function projective synchronization of unified chaotic systems with uncertain parameters," Chaos, Solitons \& Fractals, vol. 42, no. 2, pp. 12661272, 2009.

[32] P. Zhou and W. Zhu, "Function projective synchronization for fractional-order chaotic systems," Nonlinear Analysis: Real World Application, vol. 12, no. 2, pp. 811-816, 2011.

[33] K. Sebastian Sudheer and M. Sabir, "Switched modified function projective synchronization of hyperchaotic Qi system with uncertain parameters," Communications in Nonlinear Science and Numerical Simulation, vol. 15, no. 12, pp. 4058-4064, 2010.

[34] H. Du, Q. Zeng, and N. Lü, "A general method for modified function projective lag synchronization in chaotic systems," Physics Letters A, vol. 374, no. 13-14, pp. 1493-1496, 2010.

[35] T. H. Lee, J. H. Park, and S.-C. Lee, "Functional projective lag synchronization of chaotic systems with disturbances," Scientific Research and Essays, vol. 5, no. 10, pp. 1189-1193, 2010.

[36] Y. Yu and H.-X. Li, "Adaptive generalized function projective synchronization of uncertain chaotic systems," Nonlinear Analysis: Real World Application, vol. 11, no. 4, pp. 2456-2464, 2010.
[37] A. Wolf, J. B. Swift, H. L. Swinney, and J. A. Vastano, "Determining lyapunov exponents from a time series," Physica D, vol. 16, no. 3, pp. 285-317, 1985.

[38] Z. Yan, "Controlling hyperchaos in the new hyperchaotic chen system," Applied Mathematics and Computation, vol. 168, no. 2, pp. 1239-1250, 2005. 


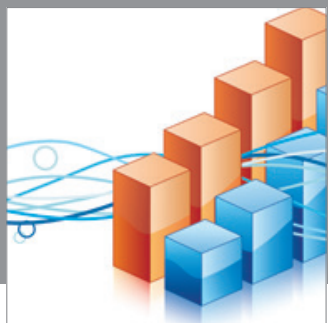

Advances in

Operations Research

mansans

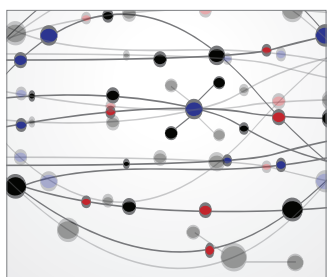

The Scientific World Journal
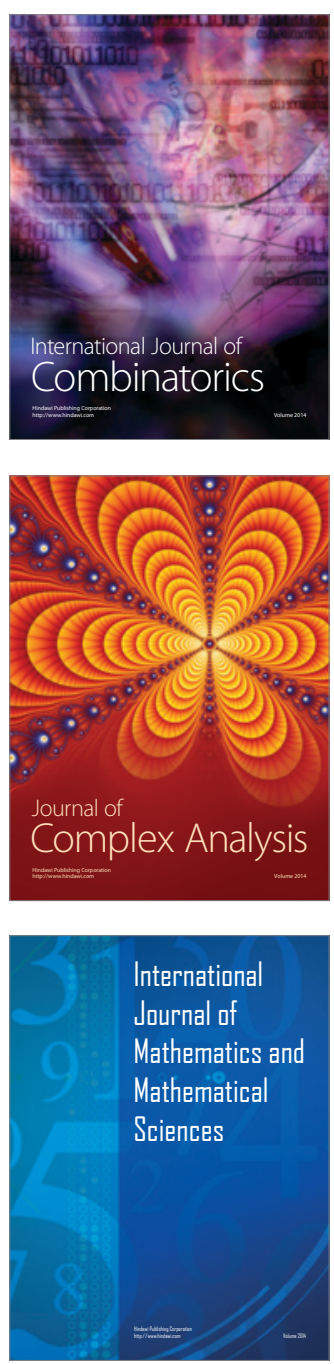
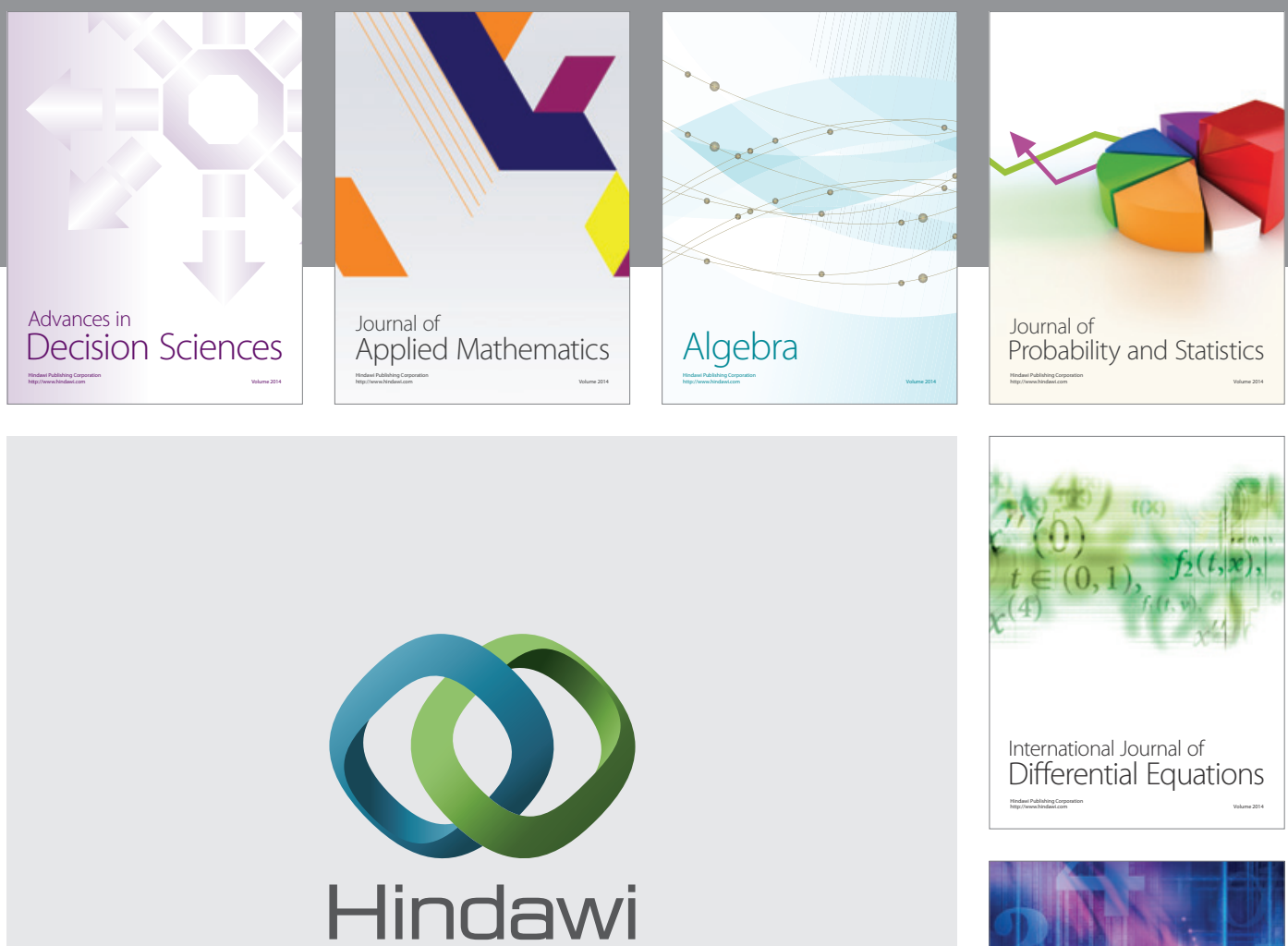

Submit your manuscripts at http://www.hindawi.com
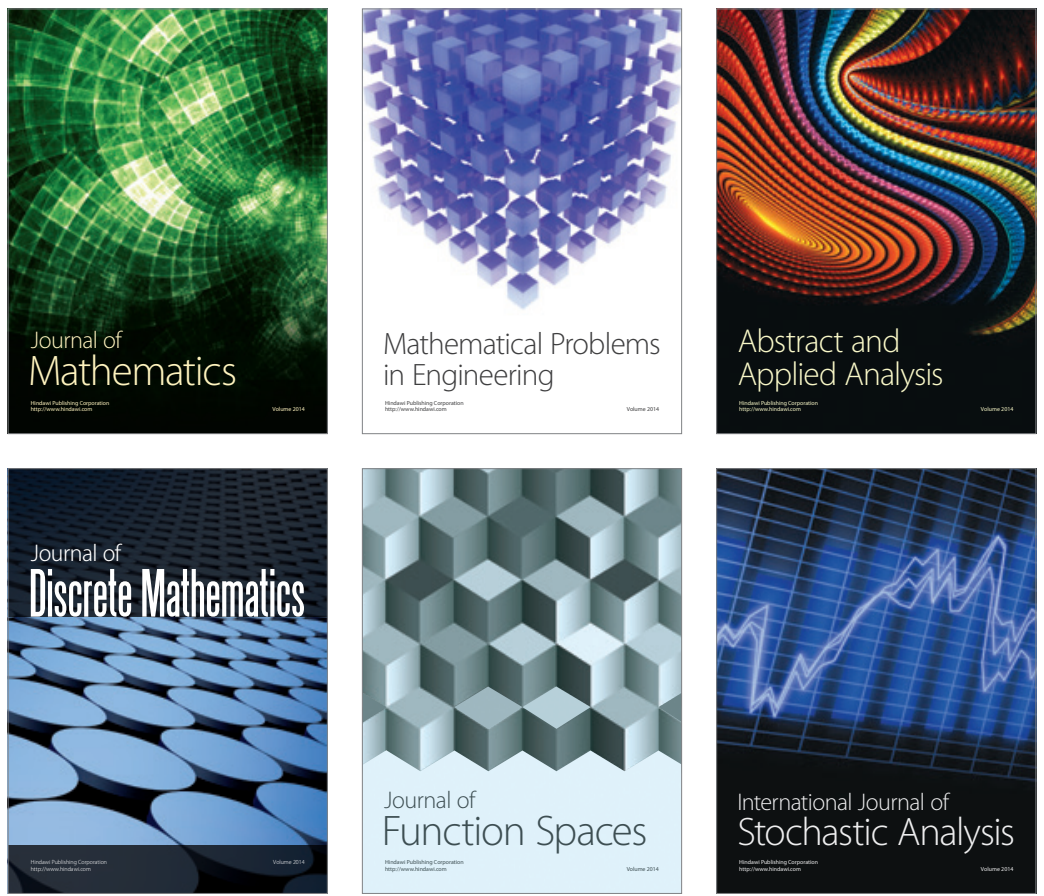

Journal of

Function Spaces

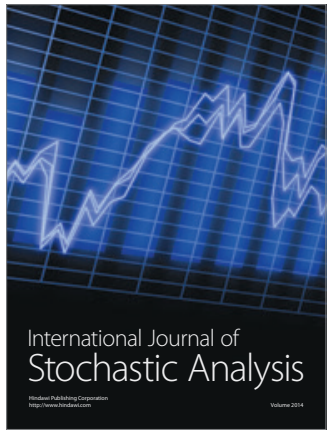

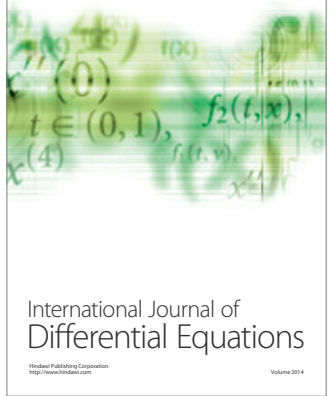
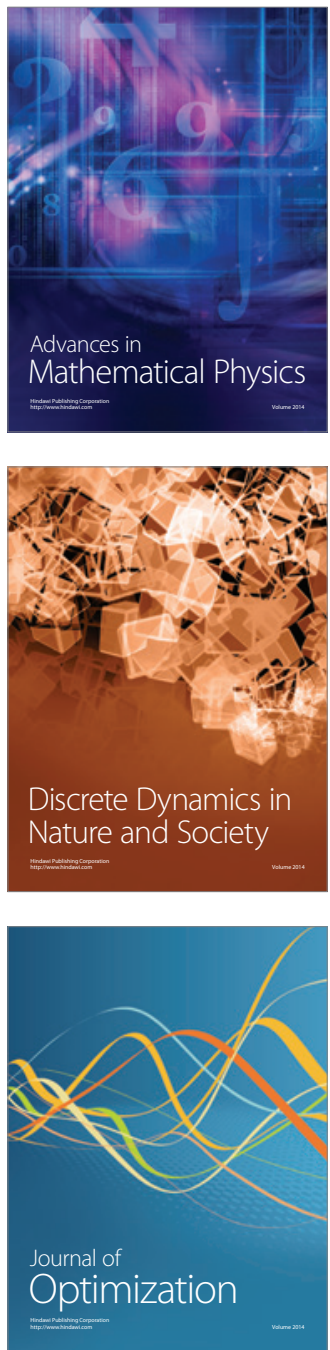